NASA/CR-2003-212089

\title{
On the Applicability of High-Frequency Approximations to Lilley's Equation
}

David W. Wundrow

Ohio Aerospace Institute, Brook Park, Ohio

Abbas Khavaran

QSS Group, Inc., Cleveland, Ohio 
Since its founding, NASA has been dedicated to the advancement of aeronautics and space science. The NASA Scientific and Technical Information (STI) Program Office plays a key part in helping NASA maintain this important role.

The NASA STI Program Office is operated by Langley Research Center, the Lead Center for NASA's scientific and technical information. The NASA STI Program Office provides access to the NASA STI Database, the largest collection of aeronautical and space science STI in the world. The Program Office is also NASA's institutional mechanism for disseminating the results of its research and development activities. These results are published by NASA in the NASA STI Report Series, which includes the following report types:

- TECHNICAL PUBLICATION. Reports of completed research or a major significant phase of research that present the results of NASA programs and include extensive data or theoretical analysis. Includes compilations of significant scientific and technical data and information deemed to be of continuing reference value. NASA's counterpart of peerreviewed formal professional papers but has less stringent limitations on manuscript length and extent of graphic presentations.

- TECHNICAL MEMORANDUM. Scientific and technical findings that are preliminary or of specialized interest, e.g., quick release reports, working papers, and bibliographies that contain minimal annotation. Does not contain extensive analysis.

- CONTRACTOR REPORT. Scientific and technical findings by NASA-sponsored contractors and grantees.
- CONFERENCE PUBLICATION. Collected papers from scientific and technical conferences, symposia, seminars, or other meetings sponsored or cosponsored by NASA.

- SPECIAL PUBLICATION. Scientific, technical, or historical information from NASA programs, projects, and missions, often concerned with subjects having substantial public interest.

- TECHNICAL TRANSLATION. Englishlanguage translations of foreign scientific and technical material pertinent to NASA's mission.

Specialized services that complement the STI Program Office's diverse offerings include creating custom thesauri, building customized databases, organizing and publishing research results ... even providing videos.

For more information about the NASA STI Program Office, see the following:

- Access the NASA STI Program Home Page at http://www.sti.nasa.gov

- E-mail your question via the Internet to help@sti.nasa.gov

- Fax your question to the NASA Access Help Desk at 301-621-0134

- Telephone the NASA Access Help Desk at 301-621-0390

- Write to: NASA Access Help Desk NASA Center for AeroSpace Information 7121 Standard Drive Hanover, MD 21076 
NASA/CR-2003-212089

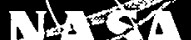

,

On the Applicability of High-Frequency Approximations to Lilley's Equation

David W. Wundrow

Ohio Aerospace Institute, Brook Park, Ohio

Abbas Khavaran

QSS Group, Inc., Cleveland, Ohio

Prepared under Contract GESS-005

National Aeronautics and

Space Administration

Glenn Research Center

January 2003 
The Propulsion and Power Program at

NASA Glenn Research Center sponsored this work.

Available from

NASA Center for Aerospace Information

7121 Standard Drive

Hanover, MD 21076
National Technical Information Service 5285 Port Royal Road Springfield, VA 22100 


\title{
On the Applicability of High-Frequency Approximations to Lilley's Equation
}

\author{
David W. Wundrow \\ Ohio Aerospace Institute \\ 22800 Cedar Point Road \\ Brook Park, Ohio 44142 \\ Email: David.W.Wundrow@grc.nasa.gov
}

\author{
Abbas Khavaran \\ QSS Group, Inc. \\ 21000 Brookpark Road \\ Brook Park, Ohio 44135 \\ Email: Abbas.Khavaran@grc.nasa.gov
}

\begin{abstract}
Three forms of the high-frequency asymptotic Green`s function for Lilley's equation are reviewed and compared to the exact solution over a wide range of Strouhal numbers. The asymmetric approximation, which applies to sources away form the jet axis, and the quasi-symmetric approximation, which is arrived at by making a near-axis source assumption, are both obtained for parallel round jets from a formal Fourier-transform solution. The ray-theory solution, which is the only high-frequency approximation that can be applied to more general mean flows, follows from a WKB ansatz and is shown to be closely related to the asymmetric approximation. The comparisons show that the best overall prediction of the exact Green's function is given by the asymmetric approximation which remains accurate down to a Strouhal number of $1 / 2$. The close relationship between the asymmetric and ray-theory approximations suggests that the high-frequency asymptotic Green's function for more general mean flows would be similarly successful.
\end{abstract}

\section{Introduction}

The prediction of jet noise has been an area of continuous interest over the last half century and has become increasingly important in recent years due to stricter noise regulations placed on the commercial aircraft industry. The need for quieter jet engines has led to several noise abatement techniques such as the placement of tabs and chevrons at the nozzle exit in order to alter aerodynamic sound generation through enhanced mixing. Accurate and robust prediction tools are instrumental in the design of more efficient noise-suppression devices.

Noise generation in jets is generally regarded as a by-product of the unsteady features of the flow and, in many situations of practical interest, the dominant feature is turbulent mixing. Jet mixing noise emanates from both fine-scale turbulence and the unsteady motions of large-scale coherent structures. The contribution from the latter component is 
usually most important at shallow angles off the downstream axis (especially in supersonic jets) and is often successfully predicted using either an instability-wave based approach or a large eddy simulation. It is this noise source that is most directly impacted by mixing enhancement devices since they tend to breakup the large structures at the expense of creating more fine-scale turbulence.

The present investigation is concerned with the mixing noise due to fine-scale turbulence which dominates the spectra away from the downstream jet axis. This component is most often analyzed by employing the acoustic analogy and assuming that the noise generating eddies are compact and behave as convected acoustic sources. The sound field is then governed by Lilley's equation which describes the acoustic propagation on a specified mean fiow due to multipole-type sources. The source distribution is, in general, modelled using appropriate space-time correlation functions.

Solutions to Lilley's equation are typically constructed by introducing a Green's function. This allows the meanflow refraction effects to be determined independent of the source distribution and limits the empiricism inherent in the acoustic analogy to the source modelling problem. Despite the linear nature of Lilley's equation, accurate numerical determination of the associated Green's function for an arbitrary mean flow is still a major undertaking [1] and consequently much attention has been focused on the simplifications to be gained by use of high-frequency asymptotics $[2,3,4]$.

The high-frequency limit arises when the acoustic wavelength of the aerodynamic noise is much shorter than the characteristic length scale of the mean flow. For simple round jets, this is usually the case within the first several jet diameters downstream of the nozzle exit where the mixing layer is thin and the turbulence intensity is at its peak. The high-frequency noise can be further increased by the presence of mixing enhancement devices. As a result, high-frequency asymptotic approximations to the Green's function for Lilley's equation form the backbone of many jet-noise prediction schemes, eg. the so-called MGB computer code [5] and its derivatives.

For arbitrary mean flows, the high-frequency solution to Lilley's equation is described in terms of the ray-theory of acoustics [4]. However, it is often reasonable to assume, for high Reynolds-number jets of practical interest, that the mean flow is both locally parallel and axisymmetric - even jets issuing from tab and chevron nozzles are known to become axisymmetric within 4 to 5 diameters downstream of the nozzle exit. When the locally parallel and axisymmetric assumption is made, three different closed-form expressions for the high-frequency Green's function for Lilley's equation are found in the literature. Goldstein [6] developed an approximation for the Green's function by restricting attention to sources located several acoustic wavelengths off the jet axis and determining the high-frequency asymptotics of the formal Fourier-transform solution available for parallel round jets. Balsa $[2,5]$ also obtained an expression for the high-frequency Green's function from the formal Fourier-transform solution but did so by assuming that the source lies near the jet centerline. Finally, Goldstein [3] presented a closed-form ray-theory solution for parallel round jets.

The goal of limiting the empiricism in jet-noise prediction schemes to the source modelling problem will be achieved only when the high-frequency asymptotics provide an accurate approximation of the exact Lilley's equation Green's function. Adequate agreement between the exact source directivity and a high-frequency approximation for Strouhal numbers as small as one has been demonstrated in some limited circumstances by Tester and Morfey [7] using a ray-theory solution for polar angles outside the zone of silence of a round jet and by Scott [8] for a two-dimensional isothermal flow with a piecewise constant mean shear. The primary objective of the present paper is to determine the relative success of the above three high-frequency approximations for parallel round jets by comparing them to the exact order-one frequency solution over a wide range Strouhal numbers and far-field observation angles. In doing so, the relationships between the different solution forms will be revealed and the potential success of the high-frequency approximation for more general mean flows will be discussed.

The general problem defining the Lilley's equation Green's function for a uni-directional transversely sheared mean flow is presented in section 2 where the formal Fourier-transform solution available for parallel round jets is given. The high-frequency asymptotic behavior of that formal solution is considered in section 3 where the approximations corresponding to the analyses of Goldstein [6] and Balsa [2,5] are summarized. Appendices A and B provide the details of those analyses with the former correcting an error in the derivation of Goldstein [6]. A comparison of the two high-frequency approximations with the exact order-one frequency Green's function is given in section 4. It is shown there that the best over all prediction of the exact result is provided by the corrected expression of Goldstein which is referred to here as the asymmetric high-frequency approximation and which remains accurate down to a Strouhal number of $1 / 2$. The Balsa expression is referred to as the quasi-symmetric high-frequency approximation 
and is shown to be at its best when applied to the ring-source directivity in which case it becomes coincident with the asymmetric approximation at sufficiently large polar angles off the downstream jet axis.

The ray-theory solution, which is reviewed for a uni-directional transversely sheared mean flow in Appendix $C$, is shown to be closely related to the asymmetric high-frequency approximation in section 5 where the generalization to complex rays in the zone of silence is also considered. In section 6 , local modifications to the ray-theory solution near the caustic and branch point are constructed and a composite solution is presented. The composite ray-theory solution is shown to provide a good approximation to the asymmetric high-frequency solution over the entire range of Strouhal numbers considered and from this result it is inferred that the high-frequency approximation for more general mean flows (i.e. mean flows that are non-axisymmetric and/or non-parallel) would be reasonably accurate down to Strouhal numbers as small as $1 / 2$.

\section{Formulation}

Interest here is in the acoustic propagation on a parallel, doubly infinite jet for which the mean flow is given by

$$
u=i \bar{u}(y, z), \quad \rho=\bar{\rho}(y, z), \quad c=\bar{c}(y, z), \quad p=\text { constant }
$$

where $u, \rho, c$ and $p$ are the velocity, density, sound speed and pressure, respectively and (1) is an exact solution to the inviscid, non-heat-conducting equations of motion. The Cartesian coordinates $\boldsymbol{x}=\{x, y, z\}$ are chosen such that $x$ is aligned with the direction of the mean flow and the unit vector $i$ is in that direction. The mean-flow profiles are required to approach constant ambient values,

$$
\bar{u} \rightarrow 0, \quad \bar{\rho} \rightarrow \bar{\rho}_{\infty}, \quad \bar{c} \rightarrow \bar{c}_{\infty}
$$

as $\sqrt{y^{2}+z^{2}} \rightarrow \infty$

Assuming a calorically perfect ideal gas, the linearized equation governing the acoustic propagation on (1) is [9]

$$
\mathcal{L} \Pi=\frac{\mathrm{D}}{\mathrm{D} t}\left(\frac{\mathrm{D}^{2}}{\mathrm{D} t^{2}}-\nabla \cdot \bar{c}^{2} \nabla\right) \Pi+2 \bar{c}^{2} \nabla \bar{u} \cdot \nabla \frac{\partial}{\partial x} \Pi=\Gamma
$$

where $\Pi$ denotes the acoustic pressure fluctuation normalized by $\bar{\rho} \bar{c}^{2}$,

$$
\frac{\mathrm{D}}{\mathrm{D} t} \equiv \frac{\partial}{\partial t}+\bar{u} \frac{\partial}{\partial x}
$$

is the convective derivative relative to the mean flow and $t$ denotes the time. The term $\Gamma$ represents the acoustic source distribution and is given by

$$
\Gamma=\frac{\mathrm{D}}{\mathrm{D} t} \nabla \cdot f-2 \nabla \bar{u} \cdot \frac{\partial}{\partial x} f
$$

when this quantity is produced by a fluctuating force per unit volume. In the absence of temperature fluctuations, Lilley's equation [10] is obtained by replacing $f$ with the quadrupole source distribution $f=\nabla \cdot(v \otimes v)$ where $v$ is the velocity fluctuation relative to the mean flow and $\otimes$ denotes the tensor product.

Since (2) is linear, the solution for an arbitrary source distribution can be obtained through superposition of solutions to

$$
\mathcal{L}\left[G_{\omega}\left(\boldsymbol{x} \mid \boldsymbol{x}_{s}\right) \mathrm{e}^{-\mathrm{i} \omega t}\right]=\frac{\mathrm{D}}{\mathrm{D} t}\left[\bar{c}_{\infty}^{2} \delta\left(x-x_{s}\right) \mathrm{e}^{-\mathrm{i} \omega t}\right],
$$

where $\omega$ is the frequency, $x_{s}$ is the source position, $\delta$ is the Dirac delta function and $G_{\omega}$ denotes a reduced Green's function. It is common practice to include the convective derivative $\mathrm{D} / \mathrm{D} t$ in the inhomogeneous term of the reduced Green's function equation because doing so simplifies the subsequent computation of the acoustic field when attention is restricted to the first term in (3) - the so-called self-noise term. It should be noted however that use of (4) does not limit the form of $\Gamma$ since the reduced Green's function $\mathbb{G}_{\omega}$ corresponding to the right-hand side,

$$
\bar{c}_{\infty}^{2} \delta\left(x-x_{s}\right) \mathrm{e}^{-\mathrm{i} \omega t}
$$


is related to $G_{\omega}$ by

$$
G_{\omega}\left(\boldsymbol{x} \mid \boldsymbol{x}_{s}\right)=-\left(\mathrm{i} \omega+\bar{u}_{s} \frac{\partial}{\partial x_{s}}\right) \mathbb{G}_{\omega}\left(\boldsymbol{x} \mid \boldsymbol{x}_{s}\right),
$$

where the subscript $s$ denotes evaluation at the source position.

When the mean flow depends only on the radial coordinate in the $y-z$ plane $r \equiv \sqrt{y^{2}+z^{2}}$, a formal solution for $G_{\omega}$ can be obtained by reducing (4) to a system of linear ordinary differential equations. Following Goldstein [6], the reduced Green's function is written as

$$
G_{\omega}\left(x \mid x_{s}\right)=\frac{1}{4 \pi^{2}} \sum_{n=-\infty}^{+\infty} \mathrm{e}^{\mathrm{in}\left(\varphi-\varphi_{s}\right)} \int_{-\infty}^{+\infty} G_{n}\left(r \mid r_{s} ; \omega, k_{1}\right) \mathrm{e}^{-\mathrm{i} k_{1}\left(x-x_{s}\right)} \mathrm{d} k_{1}
$$

where $\varphi \equiv \arctan (z / y)$ is the azimuthal angle in the $y-z$ plane. The Fourier coefficients $G_{n}$ are determined by

$$
\frac{\Phi^{2}}{r} \frac{\mathrm{d}}{\mathrm{d} r}\left(\frac{r}{\Phi^{2}} \frac{\mathrm{d}}{\mathrm{d} r} G_{n}\right)+\left[k_{0}^{2}\left(\Phi^{2}-\kappa^{2}\right)-\frac{n^{2}}{r^{2}}\right] G_{n}=-\frac{\delta\left(r-r_{s}\right)}{r a^{2}},
$$

where $\Phi \equiv(1+\kappa M) / a, k_{0} \equiv \omega / \bar{c}_{\infty}, \kappa \equiv k_{1} / k_{0}, a \equiv \bar{c} / \bar{c}_{\infty}$ is the local sound speed normalized by its ambient value and $M \equiv \bar{u} / \bar{c}_{\infty}$ is the local Mach number based on the ambient speed of sound.

Equation (7) must be solved subject to the conditions that $G_{n}$ remains bounded at $r=0$ and behaves like an outgoing wave as $r \rightarrow \infty$. The solution satisfying these conditions can be expressed in terms of two linearly independent homogeneous solutions to (7), say $w_{1}$ and $w_{2}$, as follows [11]

$$
G_{n}\left(r \mid r_{s} ; \omega, k_{1}\right)=\frac{w_{1}(r \mid \kappa) w_{2}\left(r_{s} \mid \kappa\right)}{r_{s} a_{s}^{2} W\left(r_{s} \mid \kappa\right)} \text { for } \quad r>r_{s}
$$

where

$$
W(r \mid \kappa) \equiv w_{1}(r \mid \kappa) w_{2}^{\prime}(r \mid \kappa)-w_{1}^{\prime}(r \mid \kappa) w_{2}(r \mid \kappa)
$$

is the Wronskian, a prime denotes differentiation with respect to $r$ and the $w_{3}$ have been chosen such that

$$
\begin{gathered}
w_{1} \rightarrow \text { constant } \times r^{-\frac{1}{2}} \mathrm{e}^{\mathrm{i} k_{0} \sqrt{1-\kappa^{2}} r}, \quad \text { as } r \rightarrow \infty, \\
w_{2} \rightarrow \text { constant } \times r^{|n|}, \text { as } r \rightarrow 0 .
\end{gathered}
$$

Only the $r>r_{s}$ form of $G_{n}$ is given since primary interest is in the behavior of the solution in the far field.

The homogeneous solutions $w_{3}$ must be determined numerically in general. However, when the frequency is sufficiently large, the equations become simple enough to be solved analytically. The high-frequency limit has the additional benefit of 'cutting-off' any spatially growing instability waves which appear as discrete eigensolutions to (7).

\section{High-frequency, far-field approximation}

The high-frequency limit describes the situation wherein the wavelength of the acoustic field, $1 / k_{0}=\bar{c}_{\infty} / \omega$, is much shorter than the characteristic length scale of the mean flow, viz. the jet radius

$$
r_{J} \equiv \frac{1}{M_{J}} \int_{0}^{\infty} \frac{\bar{u}(r)}{\bar{c}(r)} \mathrm{d} r=\frac{1}{M_{J}} \int_{0}^{\infty} \frac{M(r)}{a(r)} \mathrm{d} r,
$$

where $M_{J}$ is a jet Mach number which is taken here to be the ratio of the axial velocity to the local speed of sound at the jet centerline. Since the mean flow is parallel in the present analysis, the streamwise wavelength $1 / k_{1}$ scales like $1 / k_{0}$ and the high-frequency limit can therefore be expressed mathematically as

$$
\frac{\omega}{\bar{c}_{\infty}}=k_{0} \rightarrow \infty, \quad \text { with } \quad \frac{k_{1}}{k_{0}}=\kappa=O(1),
$$

where it has been assumed, for simplicity, that the mean-flow quantities $M, a$ and $r_{J}$ are all order one. 
In many technological applications, one is only interested in the behavior of the acoustic field at remote distances (in terms of the characteristic mean-flow length scale) from the aerodynamic noise sources. The so-called far-field behavior is most conveniently expressed by introducing polar coordinates in the $x-r$ plane,

$$
R=\sqrt{\left(x-x_{s}\right)^{2}+\left(r-r_{s}\right)^{2}}, \quad \theta=\arccos \left(\frac{x-x_{s}}{R}\right),
$$

with the origin at the source point, and considering the limit as $R \rightarrow \infty$.

Using (9), it can be shown that the integrand in (6) has a point of stationary phase at

$$
\kappa=-\cos \theta+O\left(R^{-1}\right),
$$

as $R \rightarrow \infty$. The integral over $k_{1}$ can then be approximated using the method of stationary phase [11] with the result that

$$
\begin{aligned}
& \int_{-\infty}^{+\infty} G_{n}\left(r \mid r_{s} ; \omega, k_{1}\right) \mathrm{e}^{-\mathrm{i} k_{1}\left(x-x_{s}\right)} \mathrm{d} k_{1} \\
& \sim\left(\frac{2 \pi k_{0} \sin ^{2} \theta}{\mathrm{i} R}\right)^{\frac{1}{2}} \frac{w_{1}(r \mid-\cos \theta) w_{2}\left(r_{s} \mid-\cos \theta\right)}{r_{s} a_{s}^{2} W\left(r_{s} \mid-\cos \theta\right)} \mathrm{e}^{\mathrm{i} k_{0} R \cos ^{2} \theta},
\end{aligned}
$$

as $k_{0}, R \rightarrow \infty$.

The asymptotic approximation for $G_{\omega}$ is completed by determining the high-frequency behavior of $w_{1}$ and $w_{2}$. To facilitate this, new dependent variables $v_{1}$ and $v_{2}$ are introduced as follows

$$
w_{j}(r \mid-\cos \theta)=\frac{\Phi(r)}{\sqrt{r}} v_{\jmath}(r) \text { for } j=1,2,
$$

where now $\Phi=(1-M \cos \theta) / a$. Substituting into (7), (9) and (10) shows that the $v_{\jmath}$ must satisfy

$$
v^{\prime \prime}+\left(k_{0}^{2} Q_{n}^{2}+\mathcal{S}\right) v=0
$$

subject to

$$
\begin{gathered}
v_{1} \rightarrow \text { constant } \times \mathrm{e}^{1 k_{0} r \sin \theta}, \quad \text { as } \quad r \rightarrow \infty, \\
v_{2} \rightarrow \text { constant } \times r^{\frac{1}{2}+|n|}, \quad \text { as } \quad r \rightarrow 0,
\end{gathered}
$$

where

$$
\begin{gathered}
r Q_{n}(r) \equiv \sqrt{r^{2} q^{2}-\left(n / k_{0}\right)^{2}}, \quad q(r) \equiv \sqrt{\Phi^{2}-\cos ^{2} \theta} \\
\mathcal{S}(r) \equiv \frac{\Phi}{r}\left(\frac{r \Phi^{\prime}}{\Phi^{2}}\right)^{\prime}+\left(\frac{1}{2 r}\right)^{2}
\end{gathered}
$$

and the square roots in (16) are chosen such that they have positive imaginary parts for negative arguments. Introducing $v_{1}$ and $v_{2}$ into (12) and the result into (6) leads to

$$
G_{\omega}\left(\boldsymbol{x} \mid \boldsymbol{x}_{s}\right) \sim \frac{\mathcal{G}_{\omega}\left(\boldsymbol{x} \mid \boldsymbol{x}_{s}\right) \mathcal{R}_{\omega}\left(\boldsymbol{x} \mid \boldsymbol{x}_{s}\right)}{a_{s}\left(1-M_{s} \cos \theta\right)}
$$

as $k_{0}, R \rightarrow \infty$, where

$$
\mathcal{G}_{\omega}\left(\boldsymbol{x} \mid \boldsymbol{x}_{s}\right) \equiv \frac{\mathrm{e}^{\mathrm{i} k_{0} R}}{4 \pi R}
$$

corresponds to the reduced free-space Green's function when both the source and far-field observation points lie in the same azimuthal plane,

$$
\mathcal{R}_{\omega}\left(\boldsymbol{x} \mid \boldsymbol{x}_{s}\right) \equiv \sum_{n=-\infty}^{+\infty}\left(\frac{2 k_{0} \sin \theta}{\mathrm{i} \pi r_{s}}\right)^{\frac{1}{2}} \frac{v_{1}(r) v_{2}\left(r_{s}\right)}{V} \mathrm{e}^{\mathrm{i} n \Delta \varphi-\mathrm{i} k_{0} R \sin ^{2} \theta}
$$


$\Delta \varphi \equiv \varphi-\varphi_{s}$ and $V \equiv v_{1} v_{2}^{\prime}-v_{1}^{\prime} v_{2}$ is the Wronskian which, in view of (13), is independent of $r$.

Before the high-frequency asymptotic solutions to (13) can be constructed, the scaling of the azimuthal wavenumber $n$ with $k_{0}$ must be considered. The summation in (20) suggests the need for uniformly valid approximations to $v_{1}$ and $v_{2}$ for all $n$. However, the dominant behavior of $G_{\omega}$ in the limit as $k_{0} \rightarrow \infty$ is determined by a relatively small number of azimuthal modes centered about a critical value of $n$ and it suffices to construct asymptotic solutions to (13) based on the $k_{0}$ scaling of that critical value.

When the distance between the source and the jet axis is sufficiently large (i.e. several factors of $1 / k_{0}$ ), the resulting acoustic field is asymmetric and the critical azimuthal wavenumber behaves like the streamwise wavenumber and scales with $k_{0}$. This scaling was considered by Goldstein [6] and will be referred to as the asymmetric, high-frequency approximation. As the source moves closer to the jet axis the acoustic field becomes increasingly axisymmetric and eventually the critical value of $n$ scales like $1 / r_{J}$. The near-axis source problem was analyzed by Balsa $[2,5]$ and will be referred to here as the quasi-symmetric, high-frequency approximation.

Solutions to (13) using the asymmetric, high-frequency scaling,

$$
n=O\left(k_{0}\right) \text { as } \quad k_{0} \rightarrow \infty
$$

were constructed by Goldstein [6] however the bounded solution used in that analysis (equation (6.61) of reference [6]) is in error. The correct asymptotic solutions for $v_{1}$ and $v_{2}$ are given in Appendix A. Using those results in (20) leads to

$$
\mathcal{R}_{\omega} \sim \sum_{n=-\infty}^{+\infty}\left[\frac{2}{k_{0}} \frac{\sqrt{-\eta_{n}\left(r_{s}\right)}}{r_{s} Q_{n}\left(r_{s}\right)}\right]^{\frac{1}{2}} \operatorname{Ai}\left[\eta_{n}\left(r_{s}\right)\right] \mathrm{e}^{\mathrm{i} n \Delta \varphi+\mathrm{i} k_{0}\left(\zeta_{n}-R \sin ^{2} \theta\right)},
$$

as $k_{0}, R \rightarrow \infty$, where

$$
\eta_{n}(r) \equiv-\left[\frac{3}{2} k_{0} \zeta_{n}(r)\right]^{\frac{2}{3}}, \quad \zeta_{n}(r) \equiv \int_{r_{\delta}}^{r} Q_{n}(r) \mathrm{d} r, \quad Q_{n}^{2}\left(r_{\delta}\right)=0,
$$

Ai denotes the Airy function and the cube root in the definition of $\eta_{n}$ is taken such that $\eta_{n} \lessgtr 0$ for $r \gtrless r_{\delta}$.

Solutions to (13) using the quasi-symmetric, high-frequency scaling,

$$
n=O(1) \text { as } k_{0} \rightarrow \infty,
$$

are given in Appendix B. Substituting (59) into (20) yields

$$
\mathcal{R}_{\omega} \sim \sum_{n=-\infty}^{+\infty} \sqrt{\mathrm{i} \frac{1}{2} \pi k_{0} \xi} \mathrm{H}_{n}^{(1)}\left(k_{0} \xi\right) \mathrm{J}_{n}\left(k_{0} \xi_{s}\right) \mathrm{e}^{\mathrm{i} n \Delta \varphi-1 k_{0} R \sin ^{2} \theta},
$$

as $k_{0}, R \rightarrow \infty$, where

$$
\xi(r) \equiv \int_{0}^{r} q(r) \mathrm{d} r=\zeta_{0}(r)-\zeta_{0}(0),
$$

$\mathrm{H}_{n}^{(1)}$ and $\mathrm{J}_{n}$ denote the Hankel and Bessel functions of the first kind, respectively, and the quantity under the square root in (22) has been simplified by making the approximation $\xi_{s} \approx r_{s} q_{s}$ which was also used in reference [5] and becomes increasingly accurate as $r_{s} \rightarrow 0$.

One of the advantages of the quasi-symmetric approximation is that the sum over $n$ in (22) can be evaluated in closed form. Using Graf's addition theorem [12], one can write

$$
\sum_{n=-\infty}^{+\infty} \mathrm{H}_{n}^{(1)}\left(k_{0} \xi\right) \mathrm{J}_{n}\left(k_{0} \xi_{s}\right) \mathrm{e}^{\mathrm{i} n \Delta \varphi}=\mathrm{H}_{0}^{(1)}\left(k_{0} \sqrt{\xi^{2}+\xi_{s}^{2}-2 \xi \xi_{s} \cos \Delta \varphi}\right)
$$

which, when substituted into (22), leads to

$$
\mathcal{R}_{\omega} \sim \mathrm{e}^{\mathrm{i} k_{0}\left(\xi-R \sin ^{2} \theta-\xi_{s} \cos \Delta \varphi\right)},
$$

as $k_{0}, R \rightarrow \infty$, where $\mathrm{H}_{0}^{(1)}$ has been replaced by its large argument behavior since $\xi \rightarrow \infty$ in the limit $R \rightarrow \infty$. 


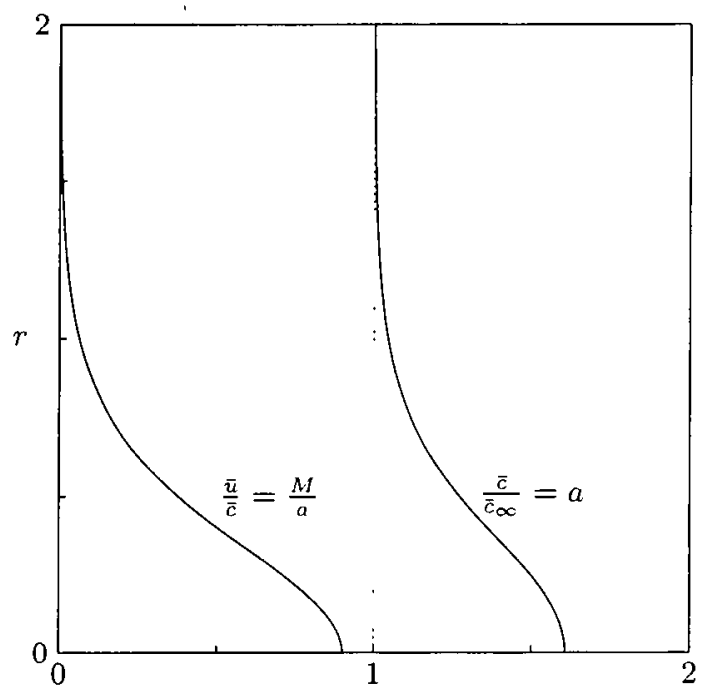

Figure 1. Mean-flow profiles (25) and (28) with $M_{J}=0.9$ and $T_{R}=3$.

\section{Comparison of exact and asymptotic solutions}

Comparisons of the high-frequency, far-field approximations to the exact solution for the reduced Green 's function are most easily carried out in terms of $\mathbb{G}_{\omega}$. It follows from (5) and (18) that

$$
\mathbb{G}_{\omega}\left(\boldsymbol{x} \mid \boldsymbol{x}_{s}\right) \sim \frac{\mathrm{i} G_{\omega}\left(\boldsymbol{x} \mid \boldsymbol{x}_{s}\right)}{\omega\left(1-M_{s} \cos \theta\right)} \sim \frac{\mathrm{i} \mathcal{G}_{\omega}\left(\boldsymbol{x} \mid \boldsymbol{x}_{s}\right) \mathcal{R}_{\omega}\left(\boldsymbol{x} \mid \boldsymbol{x}_{s}\right)}{\omega a_{s}\left(1-M_{s} \cos \theta\right)^{2}},
$$

as $k_{0}, R \rightarrow \infty$, where $\mathcal{R}_{\omega}$ is given by (21) or (23) for the asymmetric or quasi-symmetric approximation, respectively. The exact solution for $\mathbb{G}_{\omega}$ is obtained numerically using the adjoint Green's function scheme given by Tam and Auriault [13]. The interested reader is referred to that reference for the details.

The mean-flow Mach number profile used in the comparisons is

$$
\frac{\bar{u}(r)}{\bar{c}(r)}=\frac{M(r)}{a(r)}=M_{J} \operatorname{sech}^{2}(2 r),
$$

and it follows from (11) that the jet radius $r_{J}=1 / 2$. The profile is shown in figure 1 for a jet Mach number $M_{J}=0.9$.

Two different profiles are considered for the sound speed ratio $a$. The first corresponds to an isothermal jet which, in view of the ideal-gas result

$$
\bar{c}=\sqrt{\gamma \Re T},
$$

has a constant speed of sound and therefore

$$
a(r)=1,
$$

where $\gamma$ is the ratio of specific heats, $\Re$ is the gas constant and $T$ is the mean static temperature. The second profile is obtained from (26) and the Crocco-Busemann law and is given by

$$
a^{2}(r)=1+\left(1+\frac{\gamma-1}{2} M_{J}^{2}\right)^{\frac{1}{2}} \frac{T_{R}-1}{M_{J} \sqrt{T_{R}}} M(r)-\frac{\gamma-1}{2} M^{2}(r)
$$

where

$$
T_{R} \equiv\left(1+\frac{\gamma-1}{2} M_{J}^{2}\right) \frac{T_{J}}{T_{\infty}}
$$




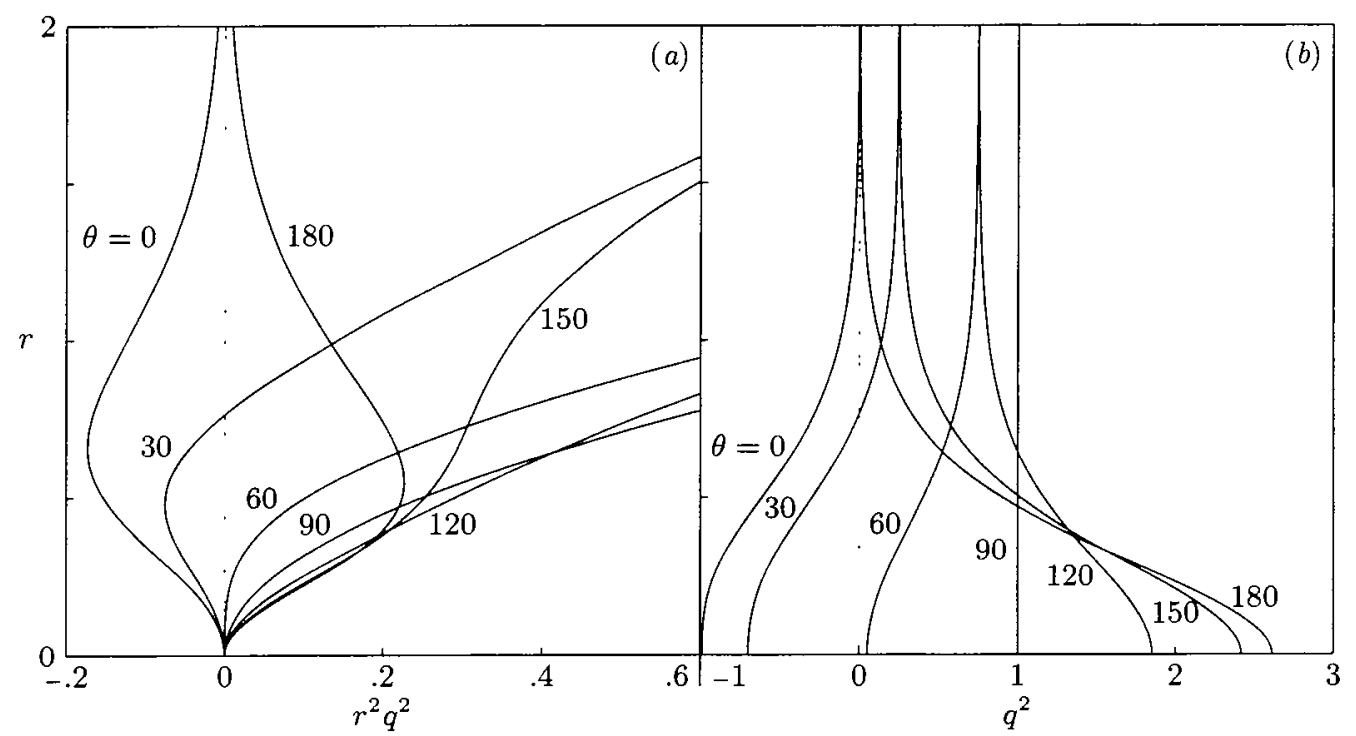

Figure 2. Turning point functions for an isothermal jet at various $\theta$.

is the ratio of the stagnation temperature at the jet centerline to the ambient temperature. In the results presented here, $\gamma=1.4, \Re=287.06 \mathrm{~J} / \mathrm{kgK}$ and $T_{\infty}=290 \mathrm{~K}$. The $a$ profile obtained from (25) and (28) with $M_{J}=0.9$ and $T_{R}=3$ is shown in figure 1 .

In deriving the asymmetric, high-frequency approximation (21), it was assumed (see Appendix A) that, for each order- $k_{0}$ value of $n,(13)$ has one simple turning point $r_{\delta}$ corresponding to a zero of $Q_{n}^{2}$. Figure 2(a) is a plot of $r^{2} q^{2}$ for various polar angles $\theta$ using (25) and (27) with $M_{J}=0.9$. Similar curves are obtained for hot jets with $a$ given by (28). Since the zeroes of $Q_{n}^{2}$ are determined by solutions to

$$
r^{2} q^{2}=\left(n / k_{0}\right)^{2}
$$

figure 2(a) shows that the turning-point assumption made in Appendix A holds for $0<\theta \leqslant 150$ provided $n / k_{0} \neq 0$. For $\theta>150$, multiple turning points are possible for certain values of $n / k_{0}$. This behavior can be accounted for by making appropriate modifications to the analysis in Appendix A, however these upstream angles are usually of no practical interest and will not be considered here.

The curves in figure 2(a) show that the turning-point assumption of Appendix $\mathrm{A}$ is violated when $n=0$ and $\theta \gtrsim 58$ in which case (29) has no real solution for $r_{\delta}$ (see also figure $2 b$ ). Since the azimuthal wavenumber scaling $n=O\left(k_{0}\right)$ clearly does not apply to the $n=0$ term in (20), it would seem that this term must be evaluated using the $n=O(1)$ scaling of the quasi-symmetric approximation. However, if the convention that $r_{\delta}=0$ when $Q_{n}^{2}$ has no zeroes is adopted (as is done for $r_{\sigma}$ in Appendix B), it can be shown that (54) and (59) agree at $n=0$ by simply replacing the functions $\mathrm{Ai}, \mathrm{H}_{0}^{(1)}$ and $\mathrm{J}_{0}$ appearing in these expressions by their large argument behaviors. The same proof also applies when $Q_{0}^{2}$ has a zero provided the simplifying assumption $r_{\delta}-r_{s} \gg k_{0}^{-2 / 3}$ of Appendix B is made. It therefore follows that the results of Appendix A remain valid even when $n=0$.

The quasi-symmetric analysis of Appendix $B$ is based on the assumption that (56) has at most one simple turning point $r_{\sigma}$ corresponding to a zero of $q^{2}$. Figure $2(b)$ is a plot of $q^{2}$ for various angles $\theta$ and the same isothermal meanflow profiles used in part $(a)$. Again, similar curves were found for heated jets. It is clear from the figure that $q^{2}$ has one simple zero for $0<\theta \lesssim 58$ and none for $\theta \gtrsim 58$. At $\theta \approx 58, q^{2}$ has a higher-order zero at $r=0$ which was not accounted for in the analysis of Appendix $B$. The effect of this omission is highly localized and, apart from a small neighborhood of $\theta \approx 58$, the turning point assumption of Appendix $B$ is satisfied for the entire range of polar angles considered here.

When comparing the exact and asymptotic solutions for $\mathbb{G}_{\omega}$, it is convenient to work in terms of the ratio $\mathbb{G}_{\omega} / \mathcal{G}_{\omega}$ since this quantity becomes independent of the radial parameter $R$ in the high-frequency, far-field limit. Figures 3-6 


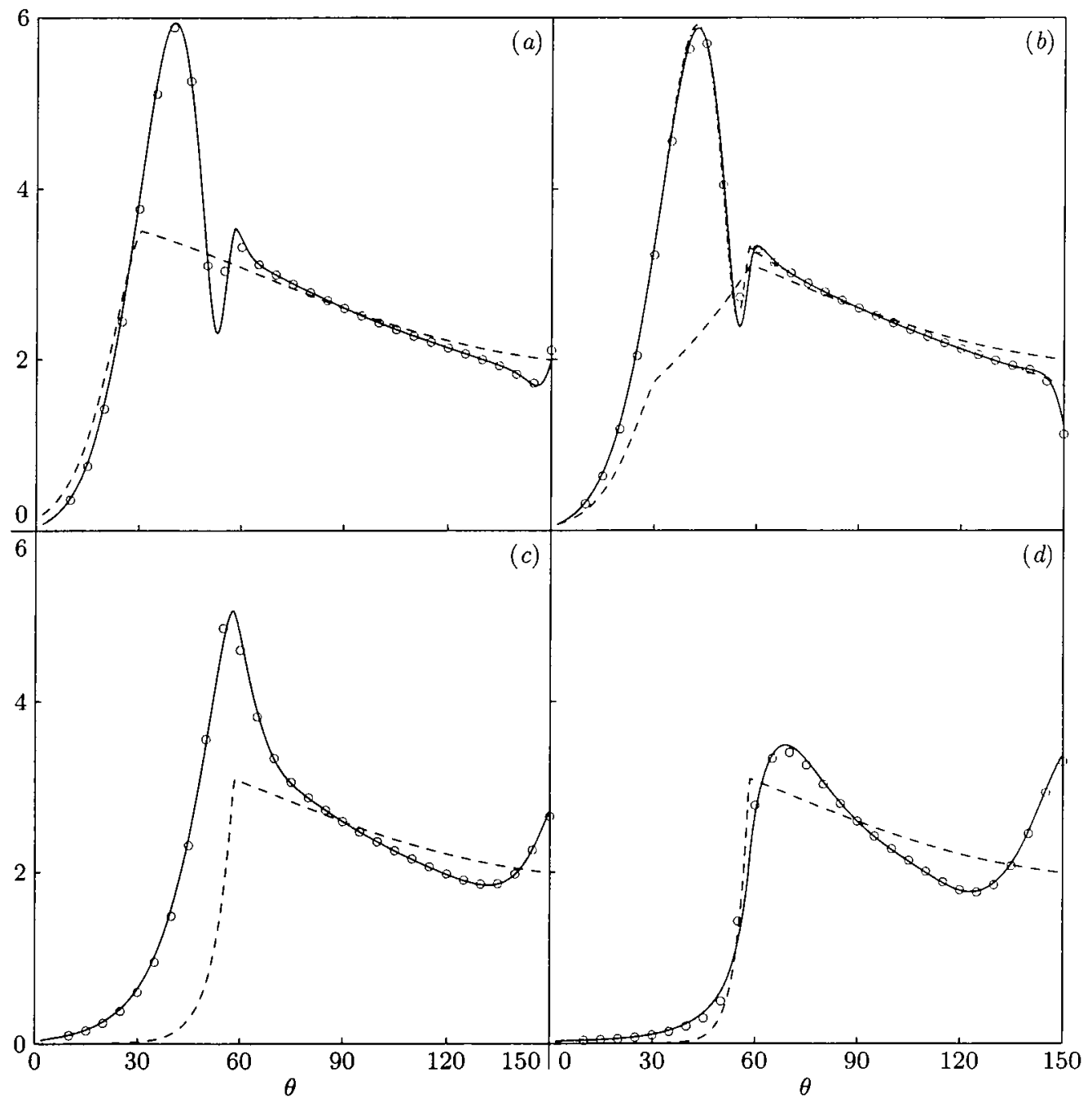

Figure 3. $\left|\mathbb{G}_{\omega} / \mathcal{G}_{\omega}\right| \times 10^{4}$ for an isothermal jet with $r_{s}=0.75$ and $S t=2$. Solid lines, asymmetric approximation; dashed lines, quasi-symmetric approximation; symbols, exact solution. (a) $\Delta \varphi=0$; (b) $\Delta \varphi=30$, dot-dashed line, composite ray solution; (c) $\Delta \varphi=90 ;($ d) $\Delta \varphi=120$.

show $\left|\mathbb{G}_{\omega} / \mathcal{G}_{\omega}\right|$ as a function of the polar angle $\theta$ for various values of the Strouhal number,

$$
S t \equiv \frac{\omega}{2 \pi} \frac{2 r_{J}}{\bar{c}_{\infty} a(0) M_{J}}=\frac{k_{0}}{\pi} \frac{r_{J}}{a(0) M_{J}} .
$$

Parts $(a-d)$ of each figure correspond to azimuthal-angle parameters $\Delta \varphi$ of $0,30,90$ and 120 . The asymmetric and quasi-symmetric approximations are indicated by the solid and dashed lines, respectively, and the symbols correspond to the exact solution. The asymmetric approximation given by (21) was evaluated by summing the convergent series in the azimuthal wavenumber $n$ from $-N$ to $+N$ where $N$ varied from 11 at $S t=2$ down to 4 at $S t=0.25$. The results in figures 3-6 were computed for a point source at $r_{s}=0.75$ using a mean flow given by (25) and (27) with $M_{J}=0.9$.

Figure 3 shows excellent agreement between the asymmetric approximation and the exact result for all $\Delta \varphi$. The level of agreement tends to diminish as $S t$ decreases but remains fairly good down to $S t=0.5$, cf. figure 5 . At the smallest Strouhal number (cf. figure 6), the asymmetric approximation, although no longer in good quantitative 


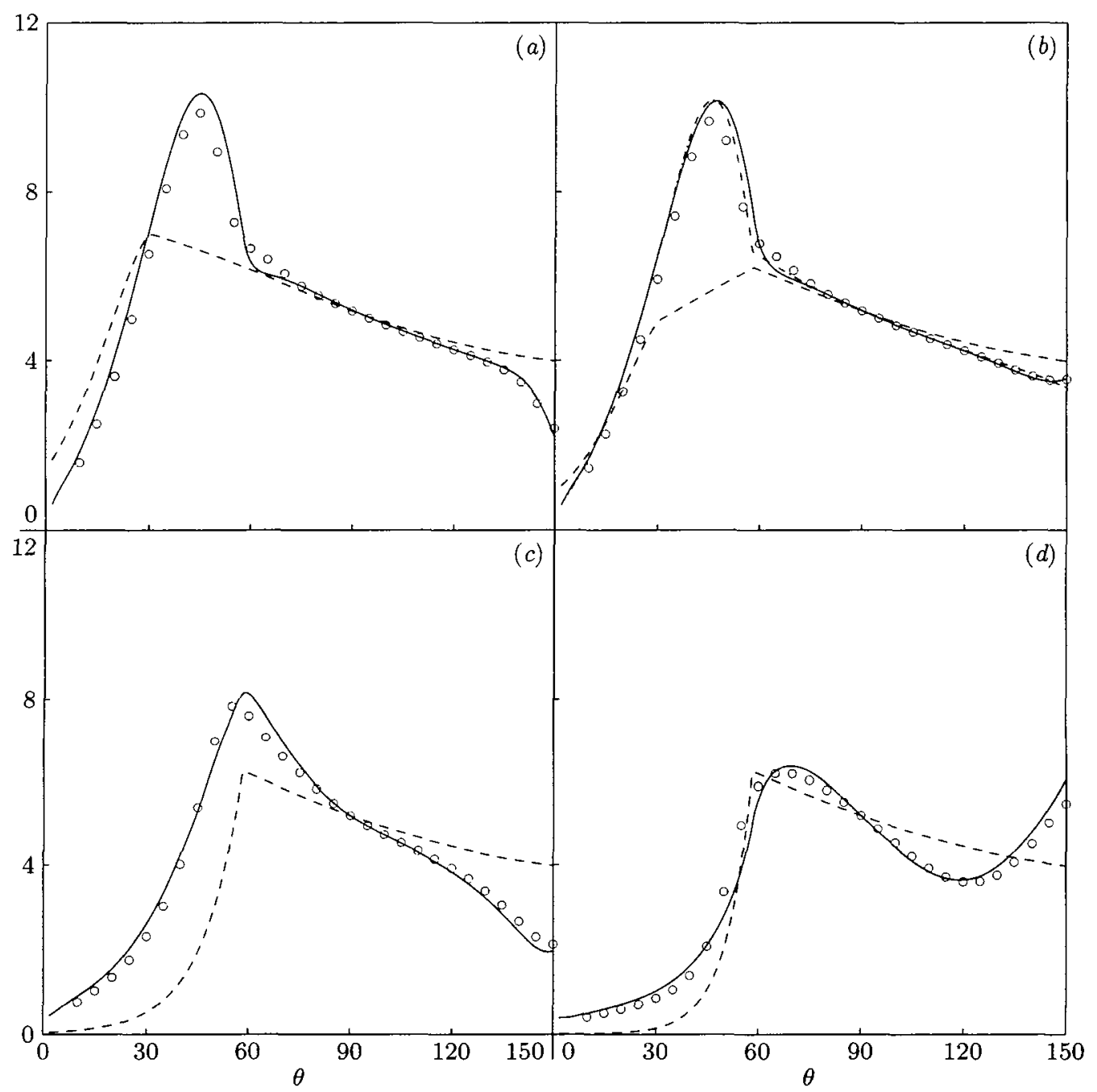

Figure 4. As figure 3 but for $S t=1$.

agreement, does still correctly predict the trends of the exact solution - a peak near $\theta=60$ with a steep drop off for $\theta<60$ and a more gradual decline for $\theta>60$.

Figures 3-6 show that, at $\theta=90$, both the asymmetric and quasi-symmetric approximations remain in near perfect agreement with the exact solution for all $S t$. At $\theta=90$, the mean-flow refraction effects for an isothermal jet vanish as can be readily verified from the expression for $\Phi$. It is interesting to note that this exceptional case is captured equally well by both high-frequency approximations despite their differences in assumed azimuthal wavenumber scaling.

Considering the quasi-symmetric approximation, the most striking feature revealed by figures 3-6 is the failure to predict the oscillatory behavior of the exact solution in the range $30<\theta<60$. This is due in large part to the assumption introduced in reference [2] (and used in Appendix B) that the no-turning-point form of $G_{\omega}$ adequately approximates the Green's function when $r_{s}>r_{\sigma}>0$. It can be seen from figure $2(b)$ that (56) has a turning point that lies between the source position $r_{s}=0.75$ and the jet axis when $30<\theta<60$. By neglecting the turning point, the quasi-symmetric approximation does not allow for ray-interference effects which are primarily responsible for the oscillations in the exact solution. The issue of interference is discussed in more detail in section 6 where the ray-theory approximation of the Green's function is considered.

The figures also show that, in the range $\theta<30$, the quasi-symmetric approximation tends to over predict $\left|\mathbb{G}_{\omega} / \mathcal{G}_{\omega}\right|$ 


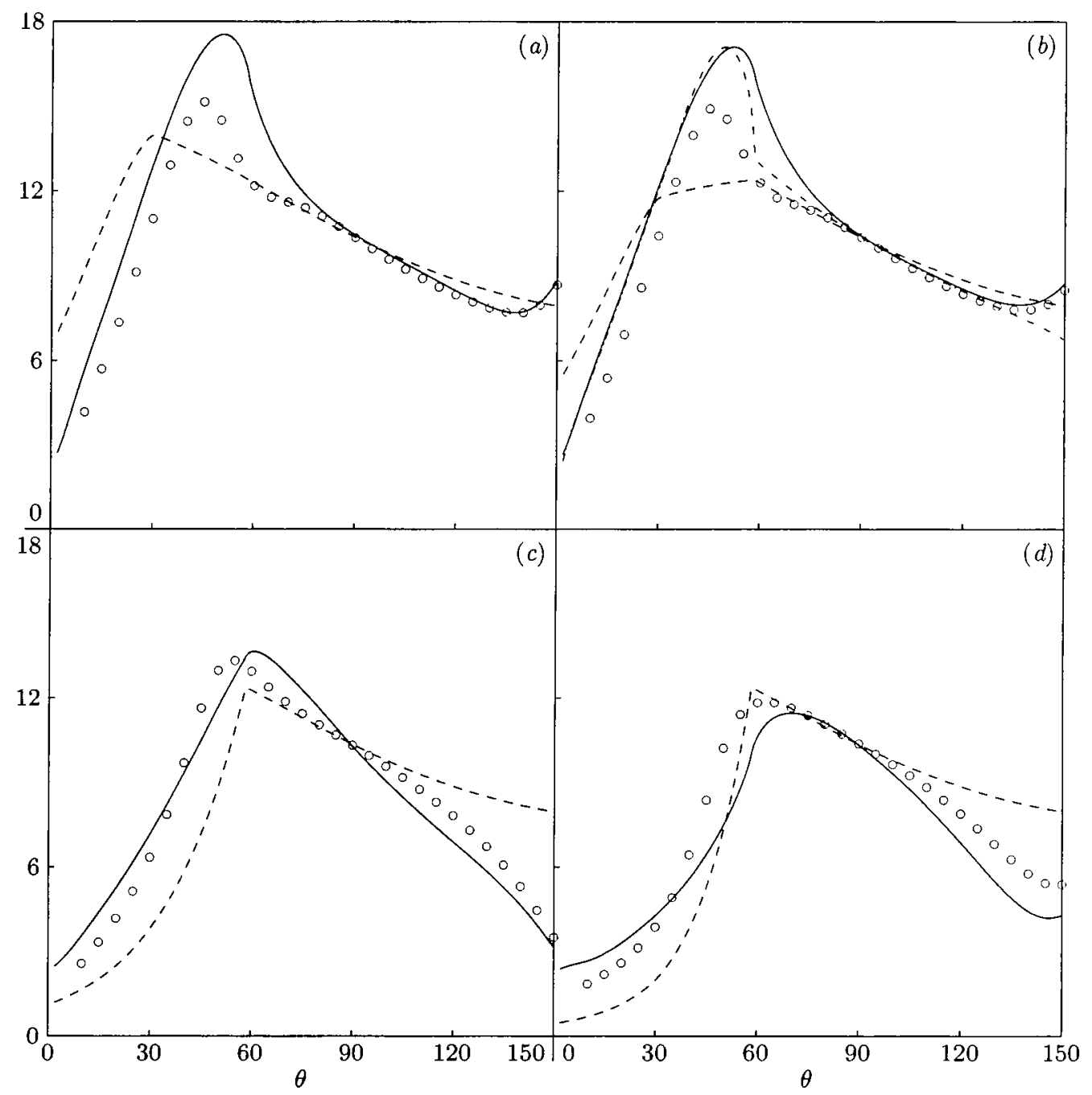

Figure 5. As figure 3 but for $S t=0.5$.

when $\Delta \varphi<90$ and under predicts this quantity when $\Delta \varphi>90$. For $\theta>60$, the agreement between the quasisymmetric approximation and the exact solution actually improves as $S t$ decreases. This surprising behavior, which was also noted by Balsa [2], is probably due to the increase in acoustic length scale with decreasing Strouhal number which, for a fixed source position, makes the solution appear more axisymmetric. It should also be noted that the ray-interference effects near $\theta=60$ diminish as $S t$ decreases and this too improves the agreement with the exact solution.

When modelling the acoustic-source distribution $\Gamma$ for use in a noise prediction scheme, it is usual to assume that the sources are sufficiently compact so that only the absolute value of $\mathbb{G}_{\omega}$ appears in the resulting formulae. Nevertheless, it may be of some interest to see how well the high-frequency, far-field approximations predict the phase of $\mathbb{G}_{\omega} / \mathcal{G}_{\omega}$. This quantity is plotted in figure 7 at $S t=1$ for various $\Delta \varphi$ and the same mean flow used for figures 36. The curves show that overall the asymmetric approximation is better than the quasi-symmetric approximation at predicting the exact result. The quasi-symmetric approximation is at its best when $\Delta \varphi<90$ and $\theta>45$.

In the case of round jets, it is often also assumed that the strength and orieritation of the sound sources that make up $\Gamma$ are independent of the azimuthal angle. The sound field emitted by such a source distribution is then axisymmetric 


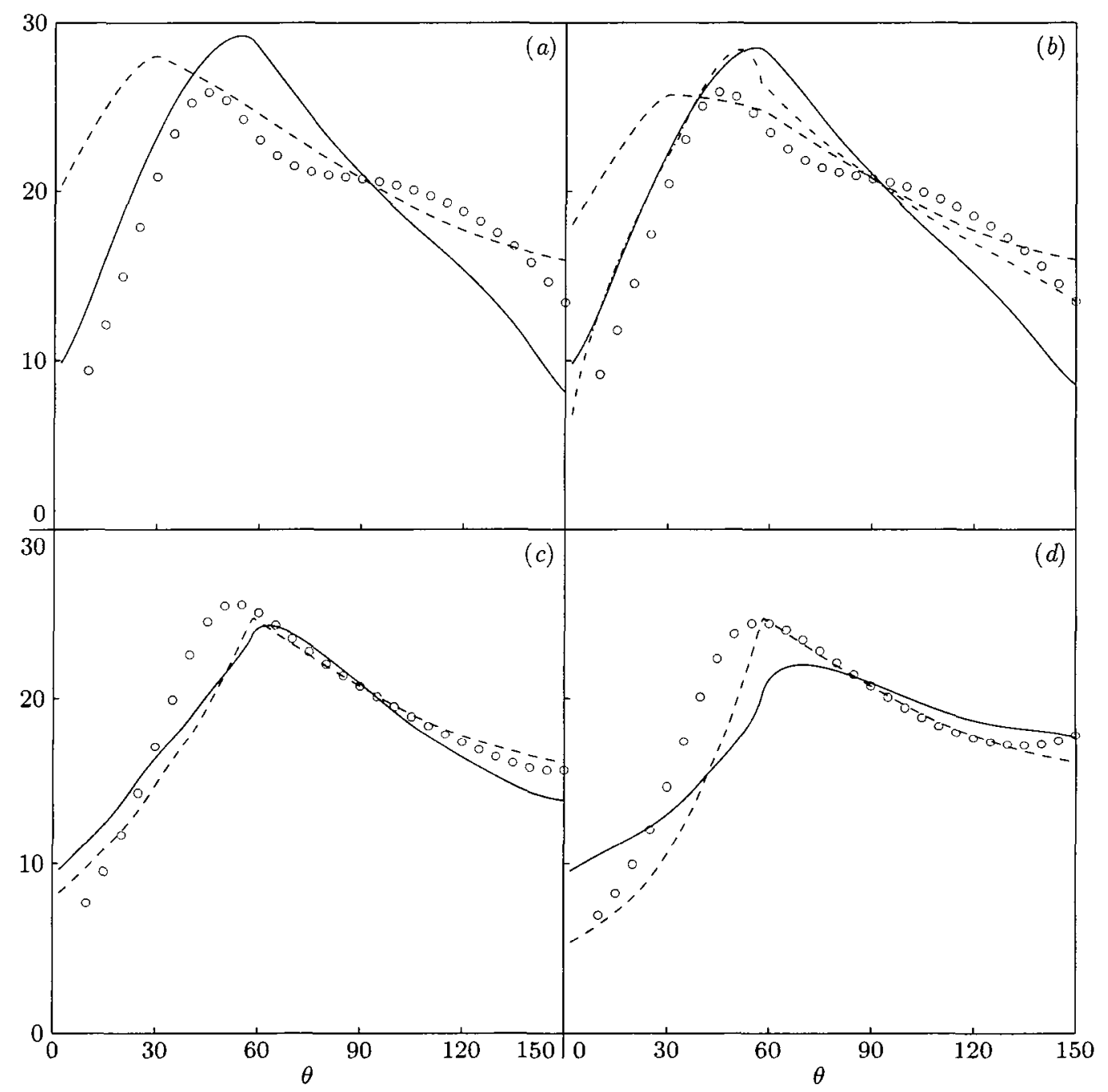

Figure 6. As figure 3 but for $S t=0.25$.

and, at fixed axial and radial positions, can be characterized by a ring-source directivity factor which is defined here as

$$
\mathcal{D} \equiv \int_{-\pi}^{+\pi}\left|\frac{\mathbb{G}_{\omega}}{\mathcal{G}_{\omega}}\right|^{2} \mathrm{~d} \varphi_{s}, \sim \frac{1}{\omega^{2} a_{s}^{2}\left(1-M_{s} \cos \theta\right)^{4}} \int_{-\pi}^{+\pi}\left|\mathcal{R}_{\omega}\right|^{2} \mathrm{~d} \varphi_{s},
$$

as $k_{0}, R \rightarrow \infty$. It follows from (21) that

$$
\int_{-\pi}^{+\pi}\left|\mathcal{R}_{\omega}\right|^{2} \mathrm{~d} \varphi_{s} \sim \frac{4 \pi}{k_{0}} \sum_{n=-\infty}^{+\infty} \frac{\sqrt{-\eta_{n}\left(r_{s}\right)}}{r_{s} Q_{n}\left(r_{s}\right)} \mathrm{Ai}^{2}\left[\eta_{n}\left(r_{s}\right)\right],
$$

for the asymmetric approximation, and from (23) that

$$
\int_{-\pi}^{+\pi}\left|\mathcal{R}_{\omega}\right|^{2} \mathrm{~d} \varphi_{s} \sim 2 \pi \mathrm{e}^{-2 k_{0} \operatorname{Im} \xi_{\sigma}} \mathrm{I}_{0}\left(2 k_{0} \operatorname{Im} \xi_{s}\right) \approx 2 \pi \mathrm{e}^{-2 k_{0} \operatorname{Im}\left(\xi_{\sigma}-\xi_{s}\right)},
$$

for the quasi-symmetric approximation, as $k_{0}, R \rightarrow \infty$, where $\mathrm{I}_{0}$ denotes the modified Bessel function of the first kind and zero-th order and a subscript $\sigma$ indicates evaluation at the turning point $r=r_{\sigma}$. The approximation introduced 


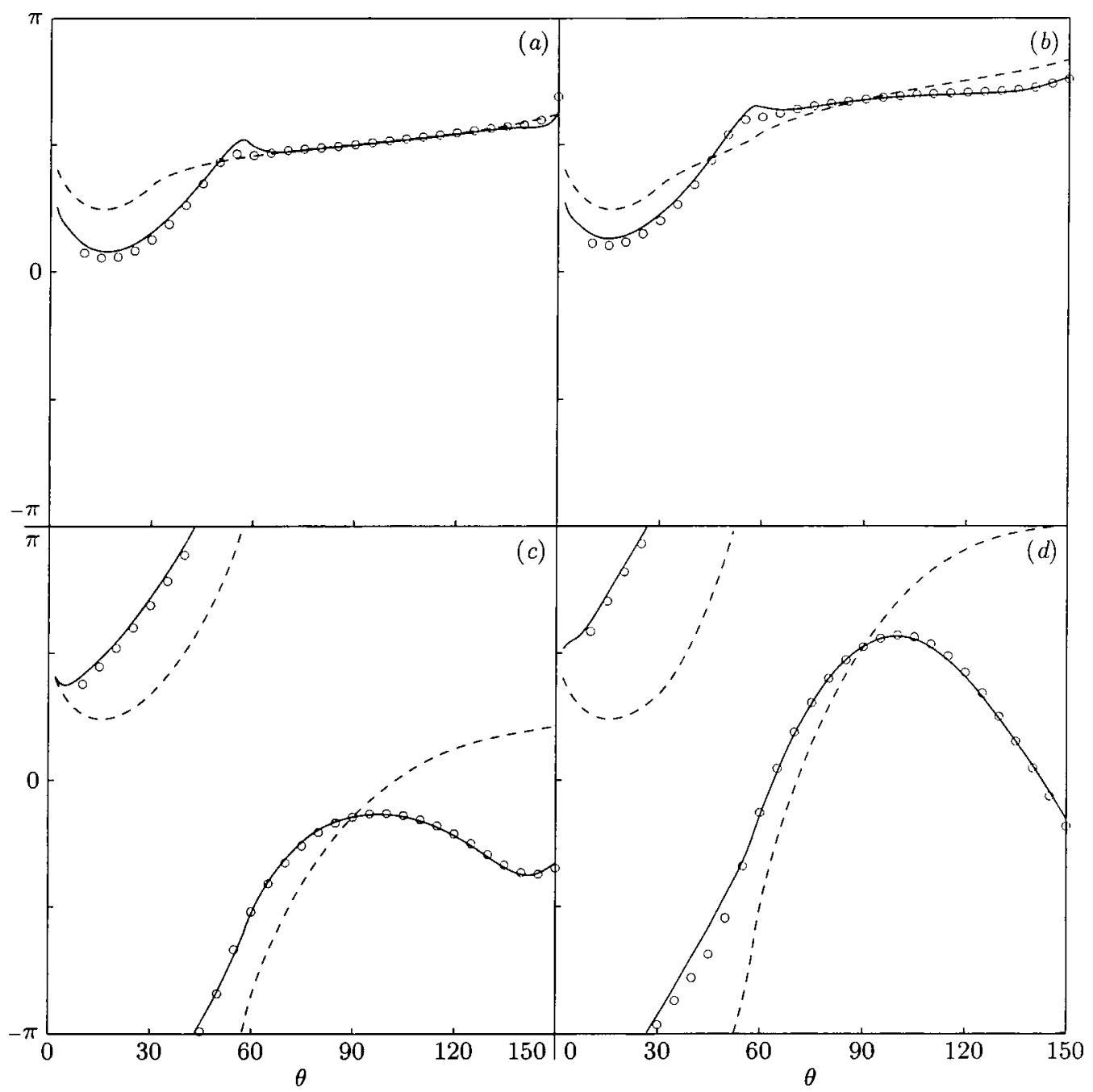

Figure 7. $\arg \left(\mathbb{G}_{\omega} / \mathcal{G}_{\omega}\right)$ for an isothermal jet with $r_{s}=0.75$ and $S t=1$. Solid lines, asymmetric approximation; dashed lines, quasi-symmetric approximation; symbols, exact solution. (a) $\Delta \varphi=0 ;$ (b) $\Delta \varphi=30 ;$ (c) $\Delta \varphi=90 ;$ (d) $\Delta \varphi=120$.

on the right-hand side of (31) corresponds to a leading-order composite expansion for the ring-source directivity and puts the quasi-symmetric approximation in agreement with the relation used in the so-called MGB computer code of reference [5].

The ring-source directivity factor is plotted as a function of polar angle $\theta$ in figures 8-11 for Strouhal numbers $S t=2,1,0.5$ and 0.25 . Parts $(a)$ and $(b)$ of each figure correspond to the mean flow given by (25) and (27) with $M_{J}=0.9$ but differing source positions, $r_{s}=0.5$ and 0.75 , respectively. Similarly, parts $(c)$ and $(d)$ show results at $r_{s}=0.5$ and 0.75 , respectively, for the heated jet given by (25) and (28) with $M_{J}=0.9$ and $T_{R}=3$.

The figures show that the agreement between the asymmetric approximation and the exact result is excellent at $S t=2$ and remains good down to $S t=0.5$. Even at $S t=0.25$, the approximation does a fair job at predicting the exact result for $\theta>60$ which corresponds to the range where the jet noise is dominated by fine-scale turbulence in many technological applications. At the two largest Strouhal numbers, the level of agreement shows no sensitivity to changes in the source radius or mean-flow temperature profile. A sensitivity to the source radius becomes apparent at the two lowest $S t$ but only in the range $\theta<60$. This may indicate an increased importance of the $n=0$ term in (30) as the source moves toward the jet center line. 


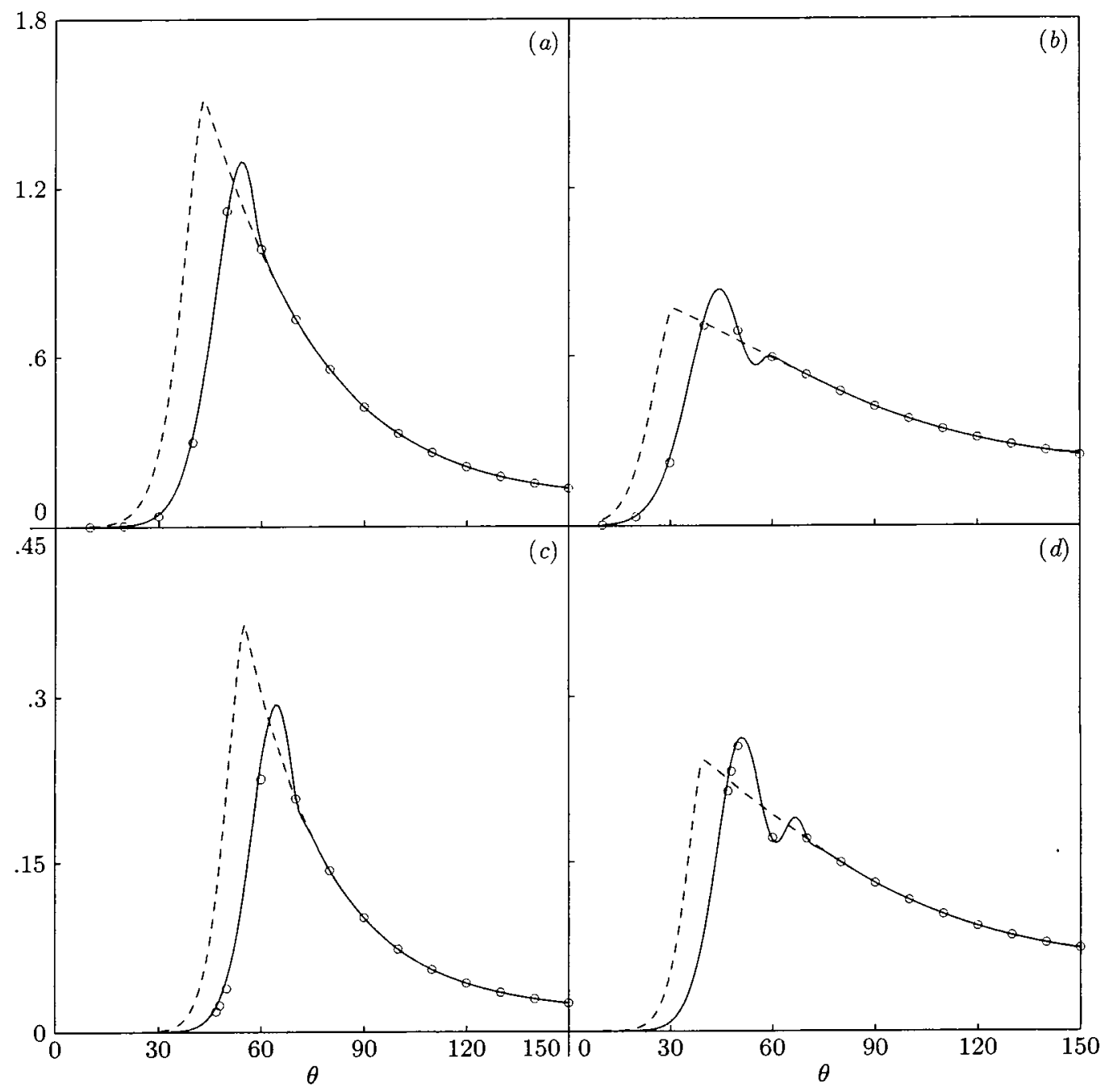

Figure 8. Ring-source directivity $\mathcal{D} \times 10^{6}$ for isothermal $(a, b)$ and heated $(c, \phi)$ jets at $S t=2$. Solid lines, asymmetric approximation; dashed lines, quasi-symmetric approximation; symbols, exact solution. $(a, c), r_{s}=0.5 ;(b, d), r_{s}=0.75$.

The agreement between the quasi-symmetric approximation and the exact solution is best when $\theta>60$ regardless of $S t$. In this range of $\theta$, the quasi-symmetric and asymmetric ring-source approximations are nearly coincident $-\mathbf{a}$ result that is not simply fortuitous. By approximating the sum in (30) with a Riemann integral [11], replacing Ai by its large (negative) argument behavior and determining the large- $k_{0}$ behavior of the resulting integral, it can be shown that the right-hand side of (30) becomes $2 \pi$ as $k_{0} \rightarrow \infty$ when $r_{s}-r_{\delta} \gg k_{0}^{-2 / 3}$ which is in exact agreement with the quasi-symmetric approximation (31).

For $\theta<60$, the quasi-symmetric approximation is never very good, primarily because of a consistent over prediction of the $\theta$ at which $\mathcal{D}$ is a maximum. This may indicate the increased importance of asymmetric effects when the Green's function problem possesses a turning point. A conjecture that is supported by the fact that the agreement in the downstream range $\theta<90$ appears to be worse for the heated jet which, it turns out, has a broader range of $\theta$ where a turning point arises in the Green's function problem. The improvement in overall agreement between the quasi-symmetric approximation and the exact result observed with decreasing $S t$ in the point-source comparison is not apparent in the ring-source comparison which suggests that the point-source observation may be strongly dependent on the azimuthal angle parameter $\Delta \varphi$. 


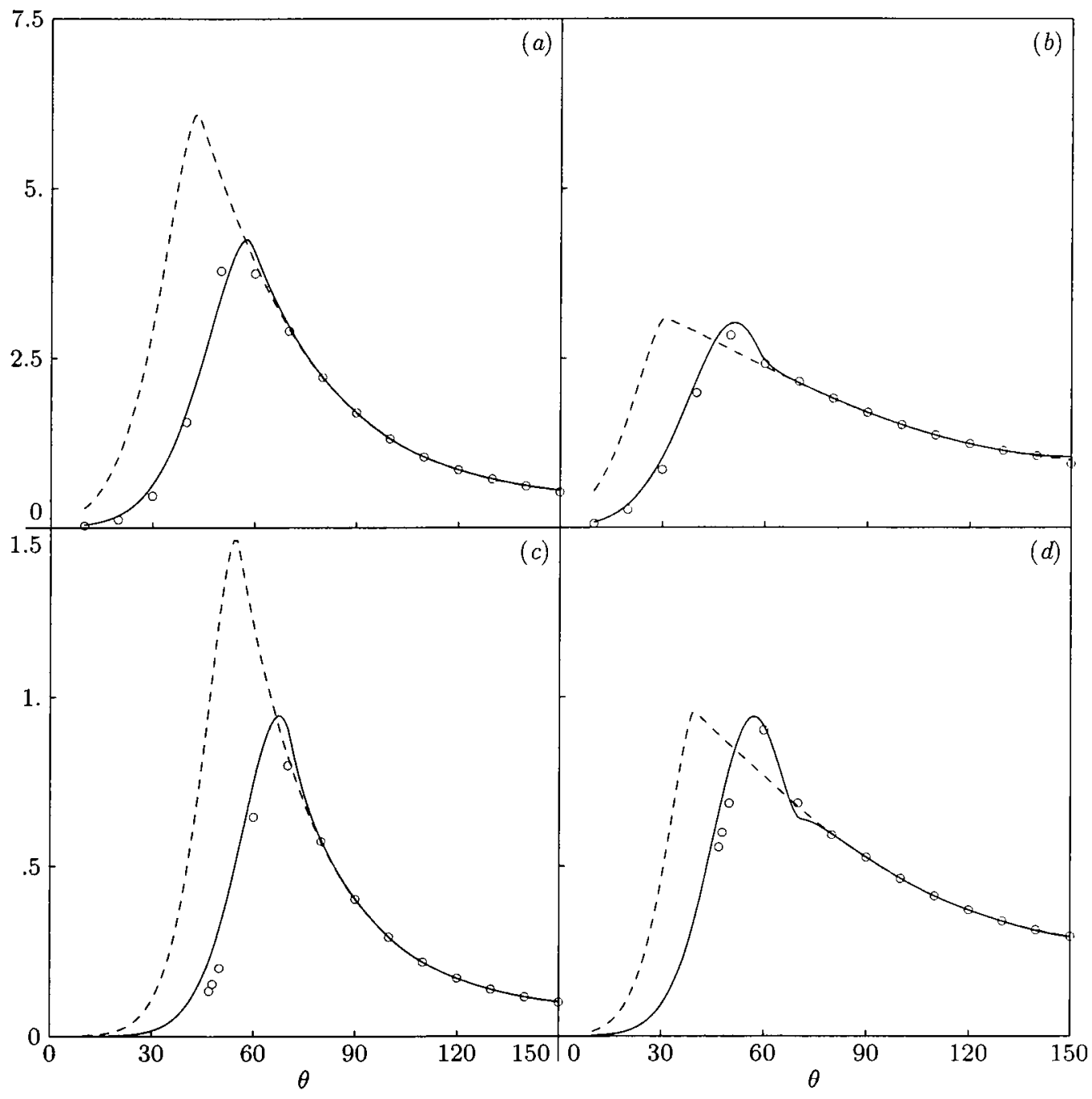

Figure 9. As figure 8 but for $S t=1$.

\section{Connection to Ray theory}

The comparisons of the previous section show that, for parallel round jets, the asymmetric, high-frequency approximation is in good agreement with the exact Lilley's equation Green's function over a wide range of Strouhal numbers. One would like to demonstrate a similar level of agreement between the exact and high-frequency asymptotic solutions for more general mean flows. Unfortunately, when the mean flow is neither axisymmetric nor parallel, the problem governing the acoustic propagation can no longer be reduced to a system of linear ordinary differential equations by Fourier analysis and an asymptotic analysis of the type described in section 3 is no longer possible. Nevertheless, analytic progress can still be made for more general mean flows by considering the high-frequency limit. The corresponding asymptotic solutions are then described in terms of the ray theory of acoustics $[3,4]$.

The ray-theory solution of (4) for the uni-directional transversely sheared mean flow (1) is summarized in Appendix $C$. It is implicitly assumed, when constructing this solution, that the length scale of the acoustic field is order $1 / k_{0}$ in all directions. Consequently, the ray-theory approximation (77) is expected to be closely related to the asymmetric approximation (21).

In order to demonstrate the connection between (21) and (77), the latter result must be specialized to an axisymmetric mean flow as done by Goldstein [3]. Introducing the mean-flow profiles $M(r)$ and $a(r)$ into (78) shows that 


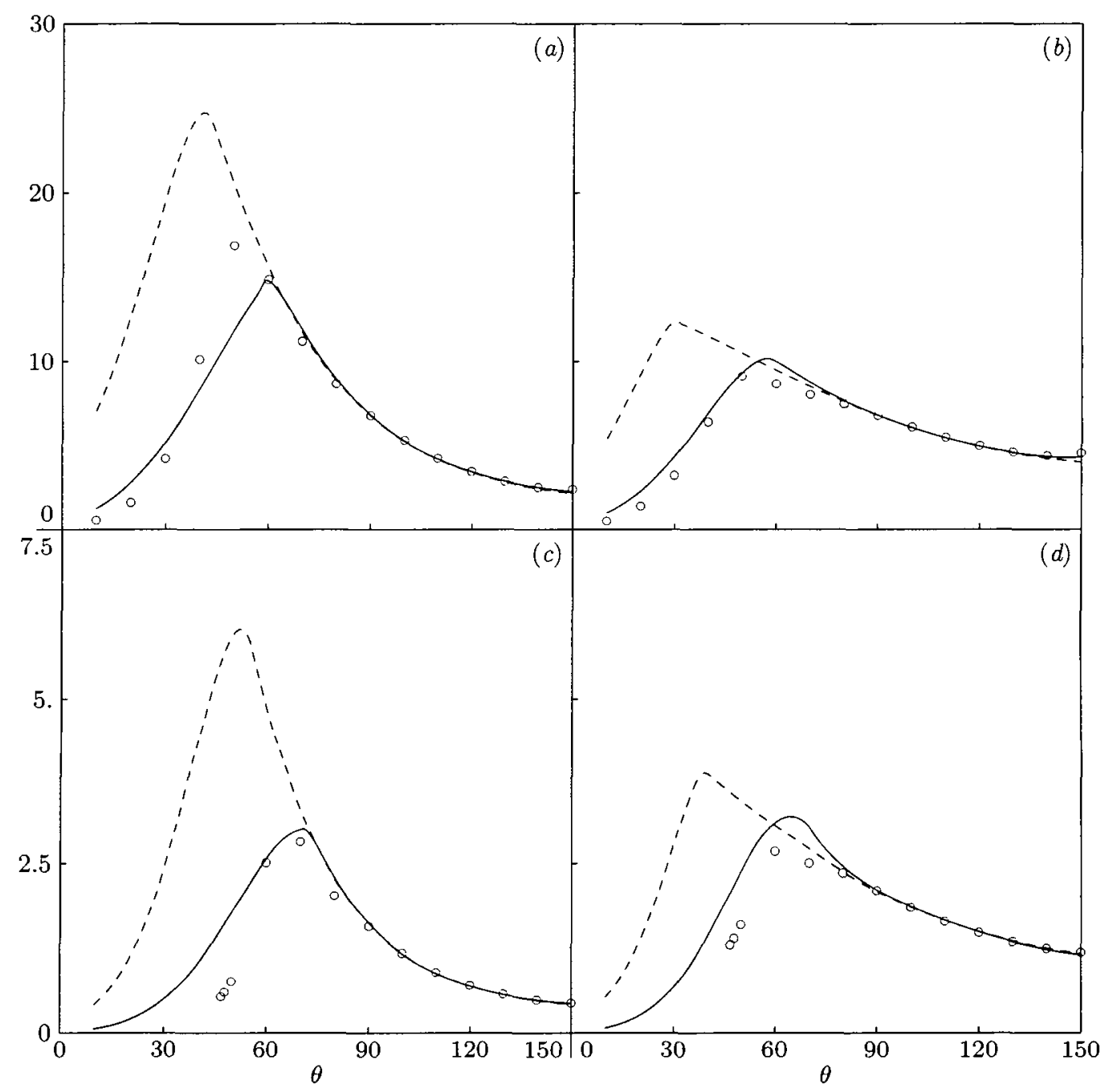

Figure 10. As figure 8 but for $S t=0.5$.

$r s^{(\varphi)}$ is constant along each ray. If (70) and (71) are then used to eliminate $\theta_{\infty}$ in favor of $\theta$ in the remaining equations, they become, to the required order of accuracy,

$$
\left.\begin{array}{c}
\dot{r}= \pm\left[Q\left(r \mid \nu_{\star}\right)+\frac{R_{\infty}-R}{R \sin \theta} \cos ^{2} \theta\right], \quad r^{2} \dot{\varphi}=\nu_{\star}, \\
\dot{S}_{\perp}=\nu_{\star} \dot{\varphi}+Q^{2}\left(r \mid \nu_{\star}\right)-\sin ^{2} \theta,
\end{array}\right\}
$$

which must be solved subject to

$$
r=r_{s}, \quad \varphi=\varphi_{s}, \quad S_{\perp}=0,
$$

at $\tau=0$, where $\nu_{\star} \equiv r_{s} q_{s} \sin \left(\lambda-\varphi_{s}\right)=r s^{(\varphi)}$,

$$
r Q\left(r \mid \nu_{\star}\right) \equiv \sqrt{r^{2} q^{2}-\nu_{\star}^{2}},
$$

a dot denotes differentiation with respect to the ray parameter $\tau$ and use has been made of (75) in arriving at the definition of $\nu_{\star}$. The order $1 / R$ term in the $\dot{r}$ equation must be retained because it leads to an order-one contribution in the far-field behavior of $S_{\perp}$. The branch cuts of $Q$ are specified below. For the present, attention will be restricted 


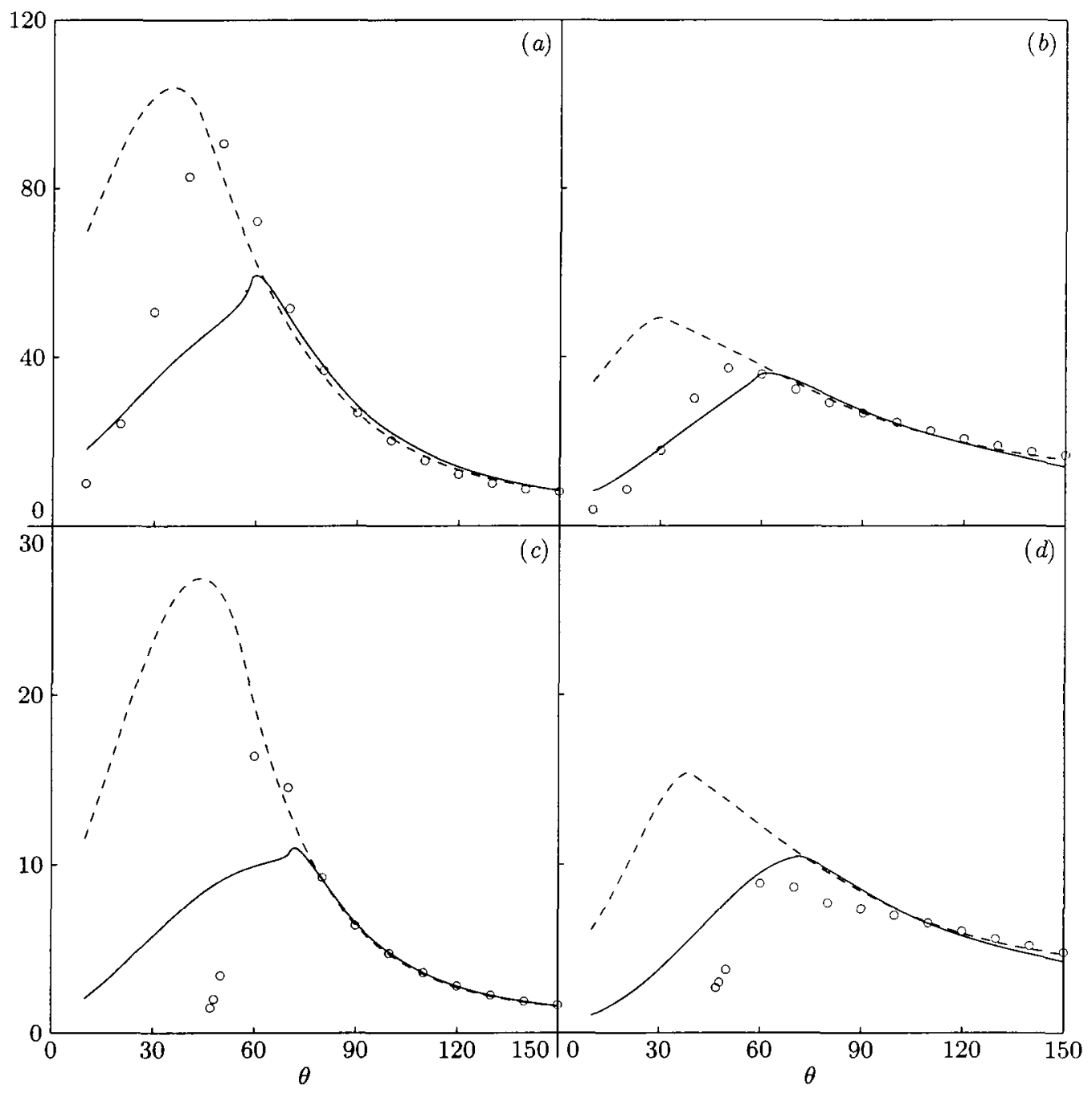

Figure 11. As figure 8 but for $S t=0.25$.

to the case where $\nu_{\star}$ is purely real which, it turns out, implies $Q^{2} \geqslant 0$ along the rays. A necessary (but not sufficient) condition for $\nu_{\star}$ to be purely real is that the source be located such that $q_{s}^{2} \geqslant 0$.

The choice of sign in the $\dot{r}$ equation of (32) is a function of the initial condition,

$$
\dot{r}=q_{s} \cos \left(\lambda-\varphi_{s}\right) \quad \text { at } \quad \tau=0,
$$

as well as the number of zeroes of $Q$ encountered along a ray trajectory $r(\tau)$. It is assumed here (in agreement with the turning-point assumptions made in Appendices $\mathrm{A}$ and $\mathrm{B}$ ) that $r Q$ is a monotonically increasing function of $r$ with at most one simple zero. $r Q$ will then be non-zero and $r$ will increase monotonically with $\tau$ for rays initially directed away from the jet centerline, i.e. $-\pi / 2<\lambda-\varphi_{s}<\pi / 2$. These solutions, for which the positive sign in (32) applies, will be referred to as direct rays. For rays initially directed toward the jet centerline, i.e. $\pi / 2<\lambda-\varphi_{s}<3 \pi / 2$, but which eventually reach the far field, $r$ initially decreases with increasing $\tau$ until $r=r_{\delta}$ where $Q$ vanishes. Once this occurs, $r$ begins to increase with $\tau$ and the sign in (32) must change from negative to positive. These solutions will be referred to as indirect rays. 
In view of the preceding discussion, the solutions to (32) can be written as

$$
\begin{gathered}
\Delta \varphi=\varphi-\varphi_{s}=\left(\int_{r_{\delta}}^{\infty} \mp \int_{r_{\delta}}^{r_{s}}\right) \frac{\nu_{\star} \mathrm{d} r}{r^{2} Q\left(r \mid \nu_{\star}\right)}, \\
S_{\perp}=\nu_{\star} \Delta \varphi+\zeta\left(r \mid \nu_{\star}\right) \mp \zeta\left(r_{s} \mid \nu_{\star}\right)-R \sin ^{2} \theta-R_{\infty}+R,
\end{gathered}
$$

where

$$
\zeta\left(r \mid \nu_{\star}\right) \equiv \int_{r_{\delta}}^{r} Q\left(r \mid \nu_{\star}\right) \mathrm{d} r, \quad Q^{2}\left(r_{\delta} \mid \nu_{\star}\right)=0,
$$

and the upper (lower) signs in (34) and (35) apply to the direct (indirect) rays. Notice that $r_{\delta}$ cancels out of both the $\Delta \varphi$ and $S_{\perp}$ solutions when the upper set of signs are chosen. Substituting these results into (77) yields

$$
\mathcal{R}_{\omega} \sim\left[ \pm r_{s} Q\left(r_{s} \mid \nu_{\star}\right) \frac{\partial \varphi}{\partial \nu_{\star}}\right]^{-\frac{1}{2}} \mathrm{e}^{\left.1 k_{0} \mid \nu_{\star} \Delta \varphi+\zeta\left(r \mid \nu_{\star}\right) \mp \zeta\left(r_{s} \mid \nu_{\star}\right)-R \sin ^{2} \theta\right]},
$$

as $k_{0}, R \rightarrow \infty$, where $\mathrm{d} \nu_{\star}= \pm r_{s} Q\left(r_{s} \mid \nu_{\star}\right) \mathrm{d} \lambda$ follows from the definition of $\nu_{\star}$.

The connection between (37) and (21) is established by showing that the former result is nothing more than the leading-order, large- $k_{0}$ approximation of the latter. This is done by using the Poisson sum formula [14] to rewrite (21) as

$$
\mathcal{R}_{\omega} \sim \sum_{m=-\infty}^{+\infty} \int_{-\infty}^{+\infty}\left[2 k_{0} \frac{\sqrt{-\eta\left(r_{s} \mid \nu\right)}}{r_{s} Q\left(r_{s} \mid \nu\right)}\right]^{\frac{1}{2}} \operatorname{Ai}\left[\eta\left(r_{s} \mid \nu\right)\right] \mathrm{e}^{\mathrm{i} k_{0}\left[\alpha_{m} \nu+\zeta(r \mid \nu)-R \sin ^{2} \theta\right]} \mathrm{d} \nu
$$

as $k_{0}, R \rightarrow \infty$, where $\alpha_{m} \equiv \Delta \varphi+2 \pi m$,

$$
\eta(r \mid \nu) \equiv-\left[\frac{3}{2} k_{0} \zeta(r \mid \nu)\right]^{\frac{2}{3}},
$$

and $Q(r \mid \nu)$ and $\zeta(r \mid \nu)$ are given by (33) and (36) with $\nu_{\star}$ replaced by $\nu$.

Since the argument of the square root in (33) can now be negative (or even complex when the $\nu$ integration of (38) is performed in the complex plane), the branch cuts of $Q$ must be made explicit. In the present context, it is clear that $Q$ is simply a generalization of the function $Q_{n}$ defined by (16) where the square root was chosen so that it has a positive imaginary part for negative (real) arguments. The appropriate generalization of this choice to arbitrary values of $\nu$ is

$$
\left.\begin{array}{c}
r Q(r \mid \nu)=\sqrt{\left|r^{2} q^{2}-\nu^{2}\right|} \mathrm{e}^{\mathrm{j} \frac{1}{2}[\arg (r q-\nu)+\arg (r q+\nu)\}} \\
\text { with } \quad-\frac{1}{2} \pi \leqslant \arg (r q \pm \nu)<\frac{3}{2} \pi
\end{array}\right\} .
$$

The branch cuts of $Q\left(r_{s} \mid \nu\right)$ in the complex $\nu$ plane are shown in figure 12. It is worth noting here that

$$
\lim _{\nu^{2} \rightarrow r_{s}^{2} q_{s}^{2}} \frac{\sqrt{-\eta\left(r_{s} \mid \nu\right)}}{r_{s} Q\left(r_{s} \mid \nu\right)}=\left[k_{0} \mathcal{P}\left(r_{s}\right)\right]^{\frac{1}{3}}
$$

where $\mathcal{P}(r) \equiv 1 / r\left(r^{2} q^{2}\right)^{\prime}$, which shows that the integrand in (38) remains bounded at the branch points of $Q\left(r_{s} \mid \nu\right)$.

The integral in (38) is evaluated asymptotically in the limit $k_{0} \rightarrow \infty$ using the method of steepest descents [11] which requires making the $k_{0}$ dependence of the integrand explicit. The Airy function Ai can be replaced with its large argument behavior along the entire real $\nu$ axis except in the small order $k_{0}^{-2 / 3}$ neighborhoods of the branch points $\pm r_{s} q_{s}$. Thus

$$
\mathcal{R}_{\omega} \sim\left\{\begin{array}{ccc}
\mathcal{R}_{\omega}^{(-)}-\mathrm{i} \mathcal{R}_{\omega}^{(+)}+\mathcal{R}_{\omega}^{(x)} & \text { for } & q_{s}^{2}>0 \\
\mathcal{R}_{\omega}^{(-)} & \text {for } & q_{s}^{2}<0
\end{array}\right.
$$

as $k_{0}, R \rightarrow \infty$, where

$$
\mathcal{R}_{\omega}^{(\mp)} \equiv \sum_{m=-\infty}^{+\infty} \int_{C^{(\mp)}}\left[\frac{\mathrm{i} k_{0}}{2 \pi r_{s} Q\left(r_{s} \mid \nu\right)}\right]^{\frac{1}{2}} \mathrm{e}^{\mathrm{i} k_{0}\left[\alpha_{m} \nu+\zeta(r \mid \nu) \mp \zeta\left(r_{s} \mid \nu\right)-R \sin ^{2} \theta\right]} \mathrm{d} \nu,
$$



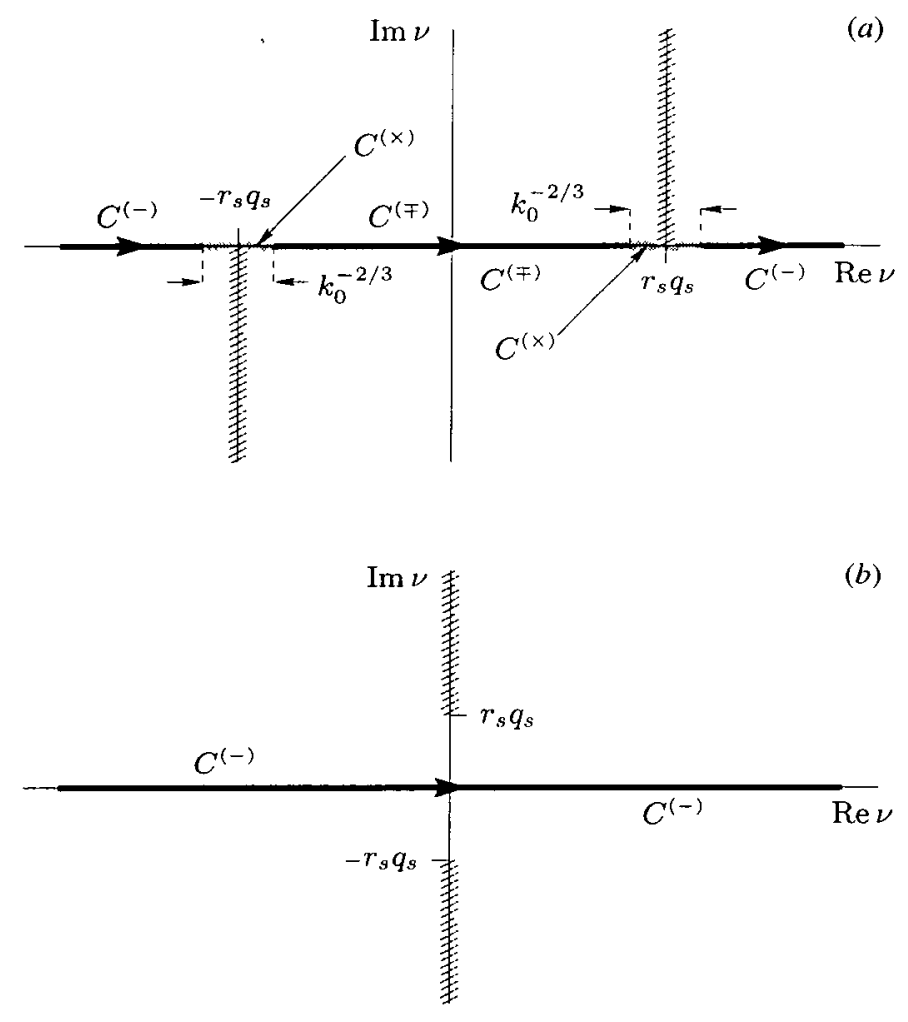

Figure 12. Branch cuts of $Q\left(r_{s} \mid \nu\right)$ in the complex $\nu$ plane. $(a) q_{s}^{2}>0, C^{(+)}$lies between $\pm r_{s} q_{s}$ where it overlaps $C^{(-)} ;(b) q_{s}^{2}<0$

and $\mathcal{R}_{\omega}^{(x)}$ is given by the right-hand side of (38) but with the integration done over the contour $C^{(x)}$. The contours $C^{(-)}, \mathscr{C}^{(+)}$and $C^{(x)}$ are shown in figure 12 .

For the present, it will be supposed that the dominant behavior of (38) is not determined by $\mathcal{R}_{\omega}^{(x)}$. This exceptional case is considered in the following section. Applying the method of steepest descents to (41) yields

$$
\mathcal{R}_{\omega}^{(\mp)} \sim \sum_{\nu_{\star}}\left[r_{s} Q\left(r_{s} \mid \nu_{\star}\right)\left|\frac{\partial \psi}{\partial \nu_{\star}}\right|\right]^{-\frac{1}{2}} \mathrm{e}^{\mathrm{i}\left(\beta+\frac{1}{4} \pi\right)} \mathrm{e}^{\mathrm{i} k_{0}\left[a_{m_{\star}} \nu_{\star}+\zeta\left(r \mid \nu_{\star}\right) \mp \zeta\left(r_{s} \mid \nu_{\star}\right)-R \sin ^{2} \theta\right]}
$$

as $k_{0} \rightarrow \infty$, where

$$
-\frac{1}{2} \pi<\beta \equiv-\arg \left[\left(\mathrm{i} \partial \psi / \partial \nu_{\star}\right)^{\frac{1}{2}}\right] \leqslant \frac{1}{2} \pi
$$

$m_{\star}$ and $\nu_{\star}$ are determined by the saddle-point condition

$$
\alpha_{m_{\star}}=\Delta \varphi+2 \pi m_{\star}=\psi\left(\nu_{\star}\right) \equiv\left(\int_{r_{\delta}}^{\infty} \mp \int_{r_{\delta}}^{r_{s}}\right) \frac{\nu_{\star} \mathrm{d} r}{r^{2} Q\left(r \mid \nu_{\star}\right)}
$$

and the $\star$ subscript is reused in order to emphasize the connection with the ray-theory solution.

It is immediately evident that the ray solution (34) and the saddle-point condition (43) are merely different versions of the same relation. The latter result determines $\nu_{\star}$ as a, possibly multi-valued, function of $\Delta \varphi$. Multiple solutions for $\nu_{\star}$ at a fixed $\Delta \varphi$ are accounted for by the summation in (42) and indicate different rays reaching the same far-field observation point. The ray solution (34) determines $\Delta \varphi$ as a single-valued function of $\nu_{\star}$ so no special treatment is needed for rays that reach the same far-field position. The $2 \pi m_{\star}$ factor in (43) allows for the possibility that $\psi$ falls outside the range $[-\pi,+\pi]$. 

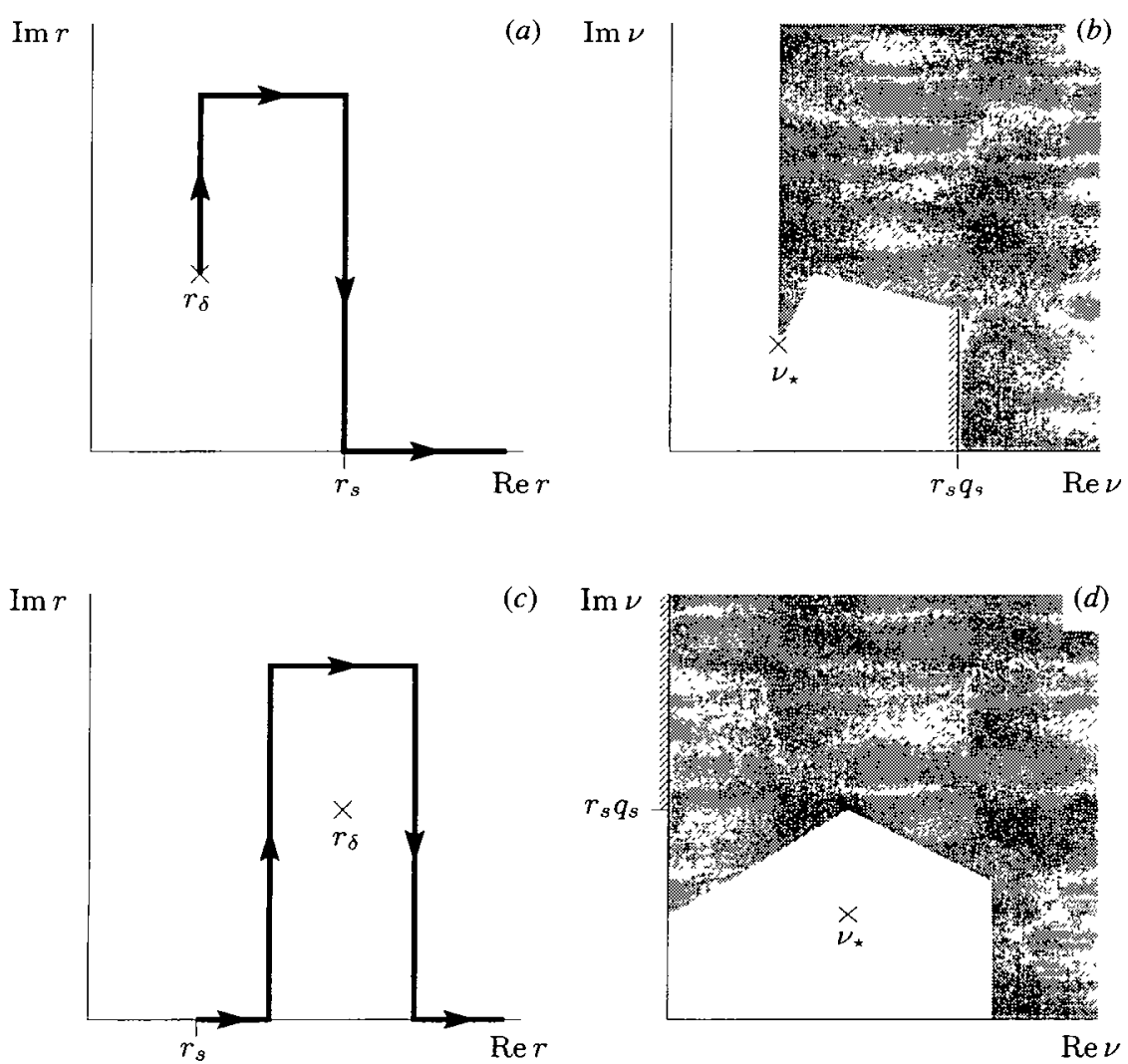

Figure 13. Contours in the complex $r$ plane $(a, c)$ and corresponding regions in the complex $\nu$ plane $(b, d)$. Shaded areas indicate location of branch cuts of $Q(r \mid \nu)$ along indicated $r$ contours. $(a, b)$, pertain to $\zeta\left(r \mid \nu_{\star}\right)+\zeta\left(r_{s} \mid \nu_{\star}\right)$ when $q_{s}^{2}>0$; (c, d), pertain to $\zeta\left(r \mid \nu_{\star}\right)-\zeta\left(r_{s} \mid \nu_{\star}\right)$ when $q_{s}^{2}<0$.

Equations (34) and (43) also imply that $\partial \psi / \partial \nu_{\star}=\partial \varphi / \partial \nu_{\star}$. If $\nu_{\star}$ is purely real as assumed when deriving (37), $\psi$ is also purely real and the amplitude factor in (42) can be rewritten as

$$
\left[r_{s} Q\left(r_{s} \mid \nu_{\star}\right)\left|\frac{\partial \psi}{\partial \nu_{\star}}\right|\right]^{-\frac{1}{2}} \mathrm{e}^{\mathrm{i}\left(\beta+\frac{1}{4} \pi\right)}=\left[r_{s} Q\left(r_{s} \mid \nu_{\star}\right) \frac{\partial \psi}{\partial \nu_{\star}}\right]^{-\frac{1}{2}} .
$$

It then follows that $\mathcal{R}_{\omega}^{(-)}$corresponds to the direct-ray solution given by the upper signs in (37) and the indirect-ray solution will correspond to $-\mathrm{i} \mathcal{R}_{\omega}^{(+)}$provided

$$
\left[-r_{s} Q\left(r_{s} \mid \nu_{\star}\right) \frac{\partial \varphi}{\partial \nu_{\star}}\right]^{-\frac{1}{2}}=\left[r_{s} Q\left(r_{s} \mid \nu_{\star}\right) \frac{\partial \varphi}{\partial \nu_{\star}}\right]^{-\frac{1}{2}} \mathrm{e}^{-\mathrm{i} \frac{1}{2} \pi}
$$

The above condition removes the ambiguity in the phase of (37) when the lower signs are taken. The $-\pi / 2$ phase shift is a consequence of the indirect ray having passed through the caustic at $r=r_{\delta}$ before reaching the far field. The shift is left undetermined in the ray-theory solution described in Appendix $\mathrm{C}$ and, in general, must be obtained through a local analysis near the caustic [15].

The above results clearly establish the connection between the asymmetric, high-frequency solution (21) and the ray-theory solution (37) when $\nu_{\star}$ is purely real. Since the location in the complex $\nu$ plane of the saddle point determined by (43) is not restricted (other than as required by the method of steepest descents), (40) and (42) show how (37) can be generalized to complex rays. The technique of applying the method of steepest descents to a classical high-frequency solution in order to guide the generalization of a ray-theory result to complex rays is well known 
$[16,17]$. Less well known are the methods for developing a complex ray theory when no classical high-frequency solution is available as, for example, in the case of the uni-directional, transversely sheared, mean flow considered in Appendix C. A survey of available approaches for doing just this is given by Chapman et al. [18].

\section{Evaluation of ray-theory solution}

In arriving at the large- $k_{0}$ approximation of $\mathcal{R}_{\omega}^{(\mp)}$ given by (42), it was implicitly assumed that the integrand in (41) can be analytically continued into the complex $\nu$ plane so as to allow integration along a contour that is (at least locally) coincident with the steepest descent paths intersecting at $\nu_{\star}$. When $\nu_{\star}$ is complex, care must be taken during the evaluation of (42) because $r_{\delta}$ is then also complex and the integration of $Q$ in the definition of $\zeta$ must be done along a contour in the complex $r$ plane. For each point along that contour, $Q$ has branch cuts in the $\nu$ plane determined by (39). The $r$-integration contour must therefore be chosen such that these branch cuts leave a region of analyticity in the complex $\nu$ plane that contains both $\nu_{\star}$ and the real axis. Figure 13 shows example contours in the complex $r$ plane used for the evaluation of $\zeta\left(r \mid \nu_{\star}\right)+\zeta\left(r_{s} \mid \nu_{\star}\right)$ and $\zeta\left(r \mid \nu_{\star}\right)-\zeta\left(r_{s} \mid \nu_{\star}\right)$ as well as the corresponding regions of analyticity in the complex $\nu$ plane.

Figure 14 contains a plot of the saddle point $\nu_{\star}$ determined from (43) as a function of polar angle $\theta$ for $\Delta \varphi=60$, $r_{s}=0.75$ and the mean flow given by (25) and (27) with $M_{J}=0.9$. The direct (indirect) ray solutions, which correspond to the upper (lower) signs in (43), are indicated by a $\triangle(\square)$. The real and imaginary parts of $\nu_{\star}$ are denoted by the open and closed symbols, respectively.

The figure shows that the complex solutions for $\nu_{\star}$ are confined to the range $\theta \lesssim 39.5$. These solutions describe the so-called zone of silence where a significant reduction in the sound radiated to the far field results from an exponential decay in $\mathcal{R}_{\omega}$. Notice that the boundary of the zone of silence does not coincide with the value $\theta \approx 30.67$ where $q_{s}^{2}=0$ (cf. figure $2 b$ ). Just outside the zone of silence is a range of $\theta$ where multiple (real) solutions for $\nu_{\star}$ are found. These multiple solutions occur when different rays reach the same far-field observation point and give rise to rayinterference effects which can be either constructive or destructive depending on the relative phases of the solutions. The ray-interference region extends to $\theta \approx 58$ beyond which $q^{2}$ has no turning points (cf. figure $2 b$ ) and only direct-ray solutions are found.

Also shown in figure 14 is the location of the branch point $r_{s} q_{s}$ along the real $\nu$ axis. The real part of $\nu_{\star}$ intersects this curve at $\theta \approx 37.5$ with the result that the solution type changes from a complex direct ray ( $\theta \lesssim 37.5$ ) to a complex indirect ray $(\theta \gtrsim 37.5)$. The change in solution type is a consequence of the phase shift in $Q\left(r_{s} \mid \nu_{\star}\right)$ that occurs as $\nu_{\star}$ crosses the branch cut issuing from $r_{s} q_{s}$ (cf. figure 12a). The asymptotic approximation (42) remains valid for $\nu_{\star}$ arbitrarily close to the branch cut (but outside the order $k_{0}^{-2 / 3}$ neighborhood of $r_{s} q_{s}$ ) because the contribution to the large- $k_{0}$ behavior of (41) obtained by deforming the $\nu$-integration contour around the branch point is of higher order.

The purely real solutions for $\nu_{\star}$ are also affected by an encounter with the $r_{s} q_{s}$ curve with the result that the indirect-ray solution $(\theta \lesssim 42)$ changes to a direct-ray solution $(\theta \gtrsim 42)$. The change in solution type occurs as $r_{\delta}$ and $r_{s}$ become coincident and corresponds to the change that occurs in (34) as $\lambda-\varphi_{\text {s }}$ passes through $\pi / 2$. The approximation of $\mathcal{R}_{\omega}$ given by (42) becomes invalid near this point because $\nu_{\star}$ moves into the order $k_{0}^{-2 / 3}$ neighborhood of a $Q\left(r_{s} \mid \nu_{\star}\right)$ branch point. The dominant behavior of (38) is then determined by $\mathcal{R}_{\omega}^{(x)}$ rather than $\mathcal{R}_{\omega}^{(-)}$or $\mathcal{R}_{\omega}^{(+)}$. The appropriate asymptotic behavior of $\mathcal{R}_{\omega}$ is found by applying the method of steepest descents to (38), where the integration is done over the contour $C^{(x)}$, with the result that

$$
\mathcal{R}_{\omega}^{(x)} \sim\left[\frac{r_{s} Q\left(r_{s} \mid \tilde{\nu}\right)}{4 \pi \sqrt{-\eta\left(r_{s} \mid \tilde{\nu}\right)}}\left|\frac{\partial \tilde{\psi}}{\partial \tilde{\nu}}\right|\right]^{-\frac{1}{2}} \operatorname{Ai}\left[\eta\left(r_{s} \mid \tilde{\nu}\right)\right] \mathrm{e}^{1 \tilde{\beta}} \mathrm{e}^{\mathrm{i} k k_{0}\left[\Delta \varphi \tilde{\nu}+\zeta(r \mid \tilde{\nu})-R \sin ^{2} \theta\right]}
$$

as $k_{0} \rightarrow \infty$, where

$$
-\frac{1}{2} \pi<\tilde{\beta} \equiv-\arg \left[(\mathrm{i} \partial \tilde{\psi} / \partial \tilde{\nu})^{\frac{1}{2}}\right] \leqslant \frac{1}{2} \pi
$$

and $\tilde{\nu}$ is determined by the saddle-point condition

$$
\Delta \varphi=\tilde{\psi}(\tilde{\nu}) \equiv \int_{r_{\delta}}^{\infty} \frac{\tilde{\nu} \mathrm{d} r}{r^{2} Q(r \mid \tilde{\nu})} .
$$

Figure 14 reveals another change in solution type which also leads to a local breakdown in the large- $k_{0}$ approximation given by $(42)$. This occurs at the zone of silence boundary $\theta \approx 39.5$ where the complex indirect-ray saddle 

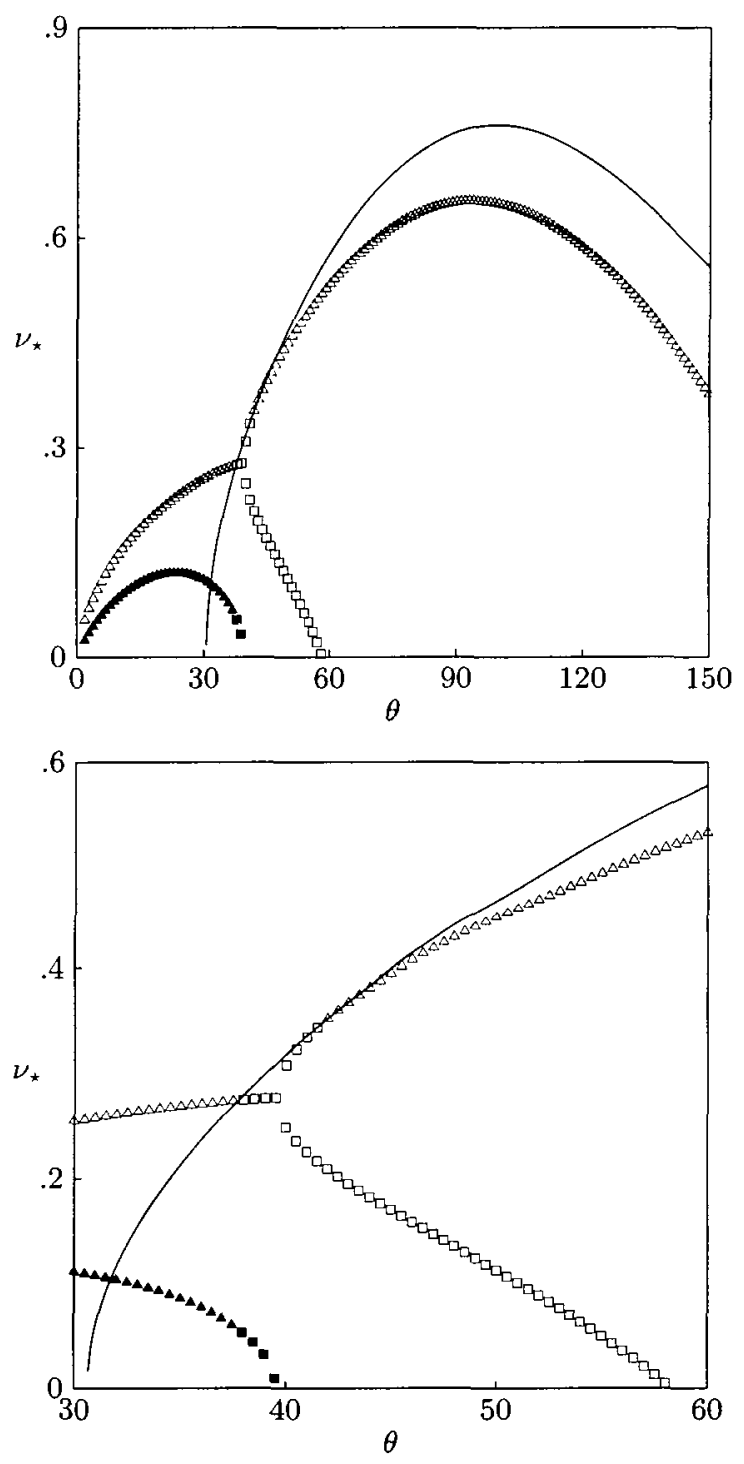

Figure 14. Saddle points $\nu_{\star}$ for an isothermal jet with $r_{s}=0.75$ and $\Delta \varphi=60$. Open symbols, $\operatorname{Re} \nu_{\star} ; \operatorname{closed} \operatorname{symbols}, \operatorname{Im} \nu_{\star} ; \Delta$, direct-ray solution; $\square$, indirect-ray solution; Solid line, $\operatorname{Re} r_{s} q_{s}$.

point changes into a pair of real indirect-ray solutions. At the point of bifurcation, $\nu_{\star}$ becomes a saddle point of higher-order for which

$$
\frac{\partial \psi}{\partial \nu_{\star}}=0
$$

with the consequence that the right-hand side of (42) becomes unbounded. Equation (47) implies a zero in the Jacobian determinant $J$ introduced in Appendix $C$ and therefore the appearance of a caustic [3], i.e. an envelope of real ray trajectories. The breakdown is restricted to indirect-ray solutions because they are the only ones that encounter a caustic before reaching the far field.

An expression for $\partial \psi / \partial \nu_{\star}$ can be derived by using (39) and the defining equation for $r_{\delta}$ to show that

$$
\frac{\partial}{\partial \nu_{\star}}\left[\frac{\nu_{\star}}{r^{2} Q\left(r \mid \nu_{\star}\right)}\right]=\frac{1+2 \nu_{\star}^{2} r \mathcal{P}^{\prime}(r)}{r^{2} Q\left(r \mid \nu_{\star}\right)}-\frac{\partial}{\partial r}\left[\frac{2 \nu_{\star}^{2} \mathcal{P}(r)}{r Q\left(r \mid \nu_{\star}\right)}\right],
$$




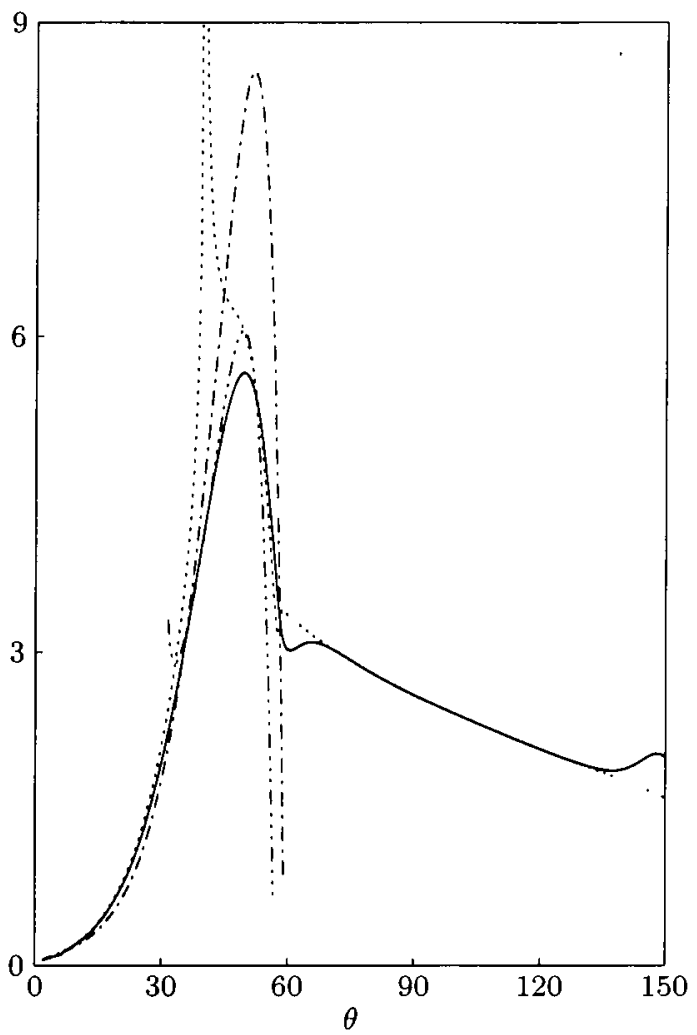

Figure 15. $\left|\mathbb{G}_{\omega} / \mathcal{G}_{\omega}\right| \times 10^{4}$ for an isothermal jet with $r_{s}=0.75, \Delta \varphi=60$ and $S t=2$. Solid line, asymmetric approximation; dotted line, ray-theory solution; dot-dashed line, near-branch-point solution; dot-dot-dot-dashed line, near-caustic solution.

$$
\frac{\partial r_{\delta}}{\partial \nu_{\star}}=2 \nu_{\star} r_{\delta} \mathcal{P}\left(r_{\delta}\right)
$$

where $\mathcal{P}(r) \equiv 1 / r\left(r^{2} q^{2}\right)^{\prime}$. Using these results when differentiating (43) with respect to $\nu_{\star}$ leads to

$$
\frac{\partial \psi}{\partial \nu_{\star}}=\left(\int_{r_{\delta}}^{\infty} \mp \int_{r_{\delta}}^{r_{s}}\right) \frac{1+2 \nu_{\star}^{2} r \mathcal{P}^{\prime}(r)}{r^{2} Q\left(r \mid \nu_{\star}\right)} \mathrm{d} r \pm \frac{2 \nu_{\star}^{2} \mathcal{P}\left(r_{s}\right)}{r_{s} Q\left(r_{s} \mid \nu_{\star}\right)},
$$

where the singularity at $r=r_{\delta}$ is integrable.

A large- $k_{0}$ approximation of $\mathcal{R}_{\omega}^{(+)}$can be constructed when the saddle point $\nu_{\star}$ approaches a zero of $\partial \psi / \partial \nu_{\star}$ by using the procedure outlined in appendix $\mathbf{G}$ of reference [19]. The integrand is expanded about the midpoint $\bar{\nu}$ rather than the saddle point $\nu_{\star}$ when applying the method of steepest descents where $\bar{\nu}$ is determined by

$$
\frac{\partial}{\partial \nu} \psi(\nu)=0, \quad \text { at } \quad \nu=\bar{\nu}
$$

Notice that, in view of (48), $\bar{\nu}$ is independent of $\Delta \varphi$. The resulting asymptotic approximation is

$$
\mathcal{R}_{\omega}^{(+)} \sim\left[\frac{\mathrm{i} 2 \pi k_{0}}{r_{s} Q\left(r_{s} \mid \bar{\nu}\right)}\right]^{\frac{1}{2}}\left[\frac{k_{0}}{2}\left|\frac{\partial^{2} \psi}{\partial \bar{\nu}^{2}}\right|\right]^{-\frac{1}{3}} \operatorname{Ai}(\bar{\eta}) \mathrm{e}^{\mathrm{i} \bar{\beta}} \mathrm{e}^{\mathrm{i} k_{0}\left[\Delta \varphi \bar{\nu}+\zeta(r \mid \bar{\nu})+\zeta\left(r_{s} \mid \bar{\nu}\right)-R \sin ^{2} \theta\right]},
$$

as $k_{0} \rightarrow \infty$, where

$$
-\frac{1}{3} \pi<\bar{\beta} \equiv-\arg \left[\left(\partial^{2} \psi / \partial \bar{\nu}^{2}\right)^{\frac{1}{3}}\right] \leqslant \frac{1}{3} \pi
$$




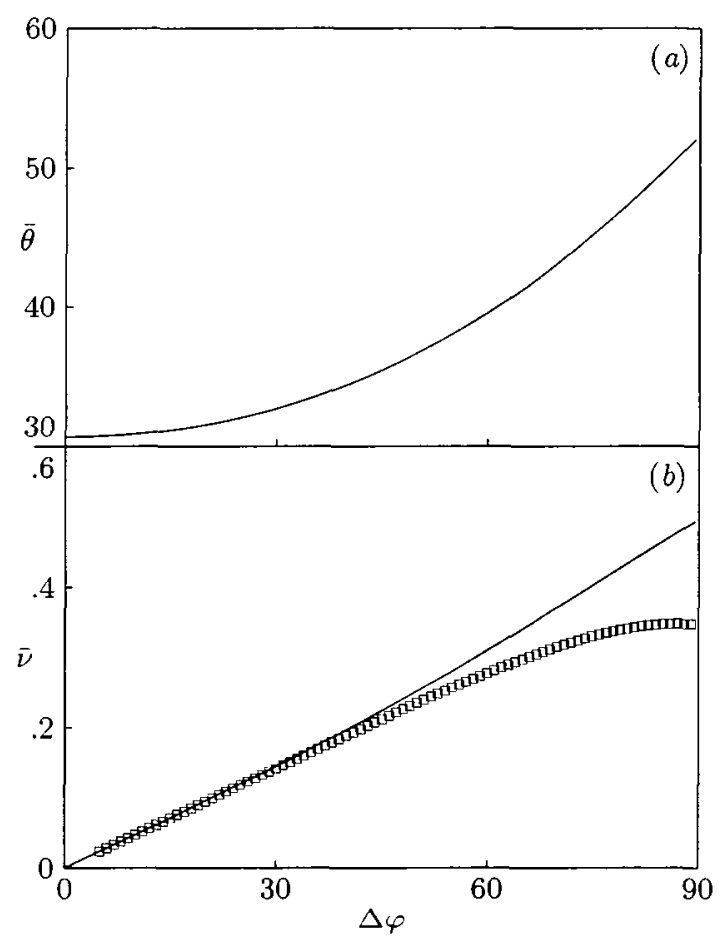

Figure 16. Various quantities evaluated at the zone of silence boundary for an isothermal jet with $r_{s}=0.75 .(b) \square$, midpoint $\bar{\nu}$; solıd line, $\operatorname{Re} r_{s} q_{s}$.

$$
\bar{\eta} \equiv-k_{0}[\psi(\bar{\nu})-\Delta \varphi]\left[\frac{k_{0}}{2}\left|\frac{\partial^{2} \psi}{\partial \bar{\nu}^{2}}\right|\right]^{-\frac{1}{3}} \mathrm{e}^{\mathrm{i} \bar{\beta}}
$$

and $\psi(\bar{\nu})$ is given by (43) with $\nu_{\star}$ replaced by $\bar{\nu}$.

Figure 15 is a plot of $\left|\mathbb{G}_{\omega} / \mathcal{G}_{\omega}\right|$ as a function of polar angle $\theta$ for Strouhal number $S t=2$ and $\Delta \varphi=60$. The solid line corresponds to the asymmetric high-frequency approximation (21). The dotted line corresponds to the ray-theory solution given by (40) and (43). Results based on the near-branch-point solution (44) and the near-caustic solution (49) are indicated by the dot-dashed and dot-dot-dot-dashed lines, respectively. The curves were computed using the same mean flow and point source location as figure 14.

Comparing the dotted and solid curves shows that the ray solution is in good agreement with the asymmetric approximation over most of the $\theta$ range including $\theta \lesssim 30$ which is well inside the zone of silence where the rays are complex. The discrepancy near $\theta=58$ is most likely due to the failure of the ray-theory solution to correctly describe the disappearance of the indirect-ray contribution (cf. figure 14). The ray-theory result could probably be improved by constructing a local solution that accounts for the higher-order zero in $Q$ that emerges for the indirect-ray solution near $\theta=58$. A similar explanation likely applies for the discrepancy near $\theta=150$ since an additional indirect-ray solution (with $m_{\star}=-1$ ) appears in the range $\theta \gtrsim 150$.

Figure 15 also shows that the ray solution breaks down at the zone of silence boundary $\theta \approx 39.5$ as expected. It is interesting to note that (42) remains bounded (although not in particularly good agreement with the asymmetric approximation) at $\theta \approx 42$ where $\nu_{\star}$ equals the branch point value $r_{s} q_{s}$ because, as can be shown from (48),

$$
\lim _{\nu_{\star}^{2} \rightarrow r_{s}^{2} q_{s}^{2}} r_{s} Q\left(r_{s} \mid \nu_{\star}\right) \frac{\partial \psi}{\partial \nu_{\star}}= \pm 2 r_{s}^{2} q_{s}^{2} \mathcal{P}\left(r_{s}\right),
$$

where again the upper (lower) sign corresponds to the direct (indirect) ray solution.

Comparing the dot-dashed, dot-dot-dot-dashed and solid curves shows that the local solutions (44) and (49) bring the ray-theory result into closer agreement with the asymmetric approximation and hence the exact solution in their 
respective regions of applicability. In the range $35<\theta<50$, the near-caustic solution (dot-dot-dot-dashed line) yields better agreement with the asymmetric approximation than does the near-branch-point solution. One might then expect that a composite solution formed from (40) and (43) together with the near-caustic solution (49) should produce good agreement with the asymmetric approximation over the ranges of $\theta$ and $\Delta \varphi$ of interest. However, as $\Delta \varphi$ decreases, the near-branch-point solution becomes the better approximation in the vicinity of the zone of silence boundary and a different approach to constructing a composite ray-theory solution will be taken here.

Figure 16(a) contains a plot of $\bar{\theta}$ as a function of $\Delta \varphi$ where $\bar{\theta}$ is defined as the value of the polar angle $\theta$ at which the indirect-ray saddle point satisfies both (43) and (47), i.e. the value at which $\nu_{\star}=\bar{\nu}$. The $\bar{\theta}$ curve therefore marks the zone of silence boundary in $\theta-\Delta \varphi$ space. As $\Delta \varphi \rightarrow 0, \bar{\theta}$ approaches 30.67 which is the value of $\theta$ at which $q_{s}^{2}$ vanishes. As $\Delta \varphi$ increases, $\bar{\theta}$ also increases indicating that the zone of silence grows as the azimuthal angle between the far-field observation point and the source point increases.

Figure 16(b) shows plots of the indirect-ray saddle point $\nu_{\star}$ (which is equivalent to $\bar{\nu}$ here) and the location of the branch point $r_{s} q_{s}$ along the real $\nu$ axis as functions of $\Delta \varphi$ at the zone of silence boundary. It is clear from the figure that $\bar{\nu}$ eventually moves into the order $k_{0}^{-2 / 3}$ neighborhood of a $Q\left(r_{s} \mid \nu_{\star}\right)$ branch point as $\Delta \varphi$ becomes sufficiently small. When this occurs, the near-caustic approximation (49) must be reworked in order to account for the presence of both a branch point and a higher-order saddle point. Rather than deriving yet another local approximation for $\mathcal{R}_{\omega}$, the approach taken here is to modify the near-branch-point approximation (44) by shifting the solution to (45) as follows

$$
\hat{\nu}=\tilde{\nu}-\left.\tilde{\nu}\right|_{\theta=\bar{\theta}}+\left.\operatorname{sign}(\tilde{\nu}) r_{s} q_{s}\right|_{\theta=\bar{\theta}} .
$$

Thus $\hat{\nu}$ coincides with the $Q\left(r_{s} \mid \nu_{\star}\right)$ branch point when $\theta=\bar{\theta}$. If the integrand in (38) is expanded about $\hat{\nu}$ rather than $\tilde{\nu}$ when the method of steepest descents is applied the following expression is obtained

$$
\mathcal{R}_{\omega}^{(x)} \sim[\text { right-hand side of (44) }] \times \exp \left[i \frac{k_{0}}{2} \frac{(\Delta \varphi-\tilde{\psi})^{2}}{\partial \tilde{\psi} / \partial \tilde{\nu}}\right],
$$

as $k_{0} \rightarrow \infty$, where $\tilde{\psi}$ is given by (46) and all occurrences of $\tilde{\nu}$ must be replaced by $\hat{\nu}$ in the above result.

The dot-dashed line in part $(b)$ of figures 3-6 corresponds to a composite ray solution for $\left|\mathbb{G}_{\omega} / \mathcal{G}_{\omega}\right|$ at $\Delta \varphi=30$ formed from outer and inner expansions given by (40) and (50), respectively. A multiplicative composite form was used when $\eta\left(r_{s} \mid \hat{\nu}\right)>0$ and an additive form otherwise [20]. The figures show that the composite ray solution does an adequate job of extending (40) through both the branch point and caustic at all St. Apart from the discrepancies near $\theta=58$ and 150 discussed above, the composite solution is in fairly good agreement with the asymmetric approximation (and hence the exact result) down to $S t=0.5$. The disagreement near $\theta=58$ and 150 tends to spread over a wider $\theta$ range as $S t$ decreases which supports the conjecture that a local large- $k_{0}$ solution could improve the ray-theory result in these regions. It is interesting to note, however, that the composite ray solution gives a better prediction of the exact result near $\theta=58$ as $S t$ decreases which may indicate that the asymmetric approximation over emphasizes the ray interference effects when $S t$ is small. At $S t=0.25$, the composite ray solution continues to accurately predict the asymmetric approximation inside the zone of silence, but, outside the zone of silence, it predicts a more gradual decline in $\left|\mathbb{G}_{\omega} / \mathcal{G}_{\omega}\right|$ with $\theta$ which puts the ray-theory result in better agreement with the exact solution.

A determination of the level of agreement between the exact and high-frequency asymptotic Lilley's equation Green's function for more general mean flows would require using the methods of reference [18] to extend the analysis of Appendix $\mathrm{C}$ to complex rays and then supplementing those results with local solutions of the sort described in reference [15] near any caustics or branch points. Such a program will not be undertaken here. Instead, it may be inferred from the success of the composite ray solution at predicting the asymmetric approximation that the level of agreement between the exact and high-frequency asymptotic solutions for more general mean flows would be similar to that shown in figures 3-11 between the exact solution and the asymmetric high-frequency approximation.

\section{Conclusions}

It has been shown that, for parallel round jets, the asymmetric high-frequency approximation, which applies to sources away from the jet axis, provides the best overall prediction of the exact Lilley's equation Green's function and remains accurate for Strouhal numbers as small as $1 / 2$. The quasi-symmetric high-frequency approximation, which 
is arrived at by making a near-axis source assumption, was found to be most successful when applied to the ringsource directivity and gives a good approximation of the exact ring-source result at all Strouhal numbers considered provided the polar angle from the downstream axis is sufficiently large. In this range of angles, an equivalence of the quasi-symmetric and asymmetric ring-source approximations was demonstrated.

The ray-theory solution was shown to be closely connected to the asymmetric high-frequency approximation and this close association was used to guide a generalization of the ray-theory result to complex rays. When combined with appropriate local solutions near the caustic and branch point, the ray-theory solution was found to be in good agreement with the asymmetric approximation and hence the exact result for the Lilley's equation Green's function. This finding was used to infer the potential for success of the high-frequency asymptotic Green's function for more general (i.e. non-axisymmetric and/or non-parallel) mean flows.

The numerical results presented here apply to stationary sources embedded in a subsonic parallel round jet and are restricted to flow situations where Lilley's equation contains at most one simple turning point. The generalization to multiple and/or higher-order turning points would require modifying the WKB analysis given in Appendix A but this is straight-forward and presents no great difficulty other than algebraic. The only impediment to applying the results of the present analysis to supersonic flows is the possibility of encountering a zero in the denominator of (24) when the source is located such that $M_{s}>1$. This singularity can however be 'removed' by the techniques developed by Ffowcs Williams [21]. Extension to sources convecting in the mean-flow direction can be made by simply introducing an appropriate Galilean transform into (4).

It was noted that development of a uniformly valid high-frequency asymptotic Green's function for more general mean flows would involve extending the ray-theory analysis of Appendix $C$ to complex rays and then supplementing those results with appropriate local solutions near any caustics or branch points. Although mathematically possible, the result of such an approach may not lend itself well to implementation in a jet-noise prediction scheme because the Green's function would then be given as a function of the initial ray direction rather than the orientation of the far-field observation point. One possible resolution to this difficulty may be the direct numerical integration of the Eikonal equation (61) and the amplitude equation (62).

\section{REFERENCES}

[1] C. K. W. TAM and N. N. PASTOUCHENKo 2002 American Institute of Aeronautics and Astronautics Journal 40, 456-464. Noise from fine-scale turbulence of nonaxisymmetric jets.

[2] T. F. BALSA 1976 Journal of Fluid Mechanics 74, 193-208. The far field of high frequency convected singularities in sheared flows, with an application to jet-noise prediction.

[3] M. E. GoldSTEIN 1982 Journal of Sound and Vibration 80, 499-522. High frequency sound emission from moving point multipole sources embedded in arbitrary transversely shear mean flows.

[4] P. A. DURBIN 1983 Journal of Sound and Vibration 91, 519-525. High frequency Green function for aerodynamic noise in moving media, Part I: general theory.

[5] R. MANI, P. R. GLIEBE and T. F. BALSA 1978 FAA-RD-76-79-II. High velocity jet noise source location and reduction.

[6] M. E. GoldstEIN 1976 Aeroacoustics. New York: McGraw-Hill.

[7] B. J. TESTER and C. L. MORFEY 1976 Journal of Sound and Vibration 46, 79-103. Developments in jet noise modelling - Theoretical predictions and comparisons with measured data.

[8] J. N. SCOTT 1979 American Institute of Aeronautics and Astronautics Journal 17, 237-244. Propagation of sound waves through a linear shear layer.

[9] M. E. GoldSTEIN 1984 Annual Review of Fluid Mechanics 16, 263-285. Aeroacoustics of turbulent shear flows.

[10] M. E. GoLDSTEIN 2001 Journal of Fluid Mechanics 443, 231-236. An exact form of Lilley's equation with a velocity quadrupole/temperature dipole source term.

[11] C. M. BENDER and S. A. ORSZAG 1978 Advance Mathematical Methods for Scientists and Engineers. New York: McGraw-Hill.

[12] M. ABRAMOWITZ and I. A. STEGUN 1964 Handbook of Mathematical Functions. Washington, D.C.: National Bureau of Standards. 
[13] C. K. W. TAM and L. AURIAULT 1998 Journal of Fluid Mechanics 370, 149-174. Mean flow refraction effects on sound radiated from localized sources in a jet.

[14] G. F. CARrier, M. KrooK and C. E. PeArson Functions of a Complex Variable. New York: Hod Books.

[15] R. N. BUCHAL and J. B. KELLER 1960 Communications on Pure and Applied Mathematics 13, 85-1 14. Boundary layer problems in diffraction theory.

[16] B. D. SECKLER and J. B. KeLLER 1959 Journal of the Acoustical Society of America 31, 192-205. Geometrical theory of diffraction in inhomogeneous media.

[17] B. D. SECKLER and J. B. KELlER 1959 Journal of the Acoustical Society of America 31, 206-216. Asymptotic theory of diffraction in inhomogeneous media.

[18] S. J. Chapman, J. M. H. Law, J. R. OCKendon and R. H. Tew 1999 Society for Industrial and Applied Mathematics Review 41, 417-509. On the theory of complex rays.

[19] D. S. JONES 1986 Acoustic and Electromagnetic Waves. Oxford: Clarendon Press.

[20] M. VAN DYKe 1975 Perturbation Methods in Fluid Mechanics. Stanford: Parabolic Press.

[21] J. E. FFowCS Williams 1963 Philosophical transactions of the Royal Society of London. Series A 255, 469503. The noise from turbulence convected at high speed.

\section{A Asymmetric, high-frequency approximation}

In this appendix, high-frequency asymptotic solutions for $v_{1}$ and $v_{2}$ are constructed using the azimuthal wavenumber scaling $n=O\left(k_{0}\right)$. The solution forms are strongly dependent on the turning points of (13) which are determined by the zeroes of $Q_{n}^{2}$. It follows from (16) that

$$
Q_{n}^{2} \rightarrow\left\{\begin{array}{c}
-\left(n / k_{0} r\right)^{2} \quad \text { as } \quad r \rightarrow 0, \\
\sin ^{2} \theta \quad \text { as } \quad r \rightarrow \infty,
\end{array}\right.
$$

and therefore that (13) always has at least one turning point with the present scaling of $n$.

For isothermal, subsonic jets with monotonically decreasing Mach number profiles, it can be shown [6] that $Q_{n}^{2}$ has at most one simple zero for $0<\theta \leqslant 90$. The situation becomes complicated for more general jet profiles and for polar angles in the range $90<\theta<180$ due to the possibility of multiple and/or higher-order turning points which then depend on the detailed shape of the mean-flow profiles. In order to keep the analysis as simple as possible, it will be assumed here (as well as in Appendix B) that (13) has at most one simple turning point. The present analysis can, if necessary, be extended to more complicated situations by making some straight-forward modifications to the results given here.

Let $r_{\delta}$ denote the single $n$-dependent turning point of (13) then the general solution

$$
v \sim\left\{\begin{array}{lr}
\left|Q_{n}\right|^{-\frac{1}{2}}\left(A^{-} \mathrm{e}^{-\mathrm{i} k_{0} \zeta_{n}}+B^{-} \mathrm{e}^{\mathrm{i} k_{0} \zeta_{n}}\right), & r_{\delta}-r \gg k_{0}^{-\frac{2}{3}}, \\
\widetilde{A} \mathrm{Ai}\left(\tilde{\eta}_{n}\right)+\widetilde{B} \mathrm{Bi}\left(\tilde{\eta}_{n}\right), & \left|r-r_{\delta}\right|=O\left(k_{0}^{-\frac{2}{3}}\right), \\
\left|Q_{n}\right|^{-\frac{1}{2}}\left(A^{+} \mathrm{e}^{\mathrm{i} k_{0} \zeta_{n}}+B^{+} \mathrm{e}^{-\mathrm{i} k_{0} \zeta_{n}}\right), & r-r_{\delta} \gg k_{0}^{-\frac{2}{3}},
\end{array}\right.
$$

as $k_{0} \rightarrow \infty$, follows from WKB theory [11], where

$$
\begin{gathered}
\tilde{\eta}_{n} \equiv \chi\left(r_{\delta}-r\right), \quad \chi \equiv\left(\left.k_{0}^{2} \frac{\mathrm{d}}{\mathrm{d} r} Q_{n}^{2}\right|_{r=r_{\delta}}\right)^{\frac{1}{3}}, \\
\zeta_{n}(r) \equiv \int_{r_{\delta}}^{r} Q_{n}(r) \mathrm{d} r, \quad Q_{n}^{2}\left(r_{\delta}\right)=0,
\end{gathered}
$$

and $\mathrm{Ai}$ and $\mathrm{Bi}$ denote Airy functions in the notation of reference [12]. The constants $A^{ \pm}, B^{ \pm}, \widetilde{A}$ and $\widetilde{B}$ are related by

$$
2 A^{-}=\left(\frac{\chi}{\pi k_{0}}\right)^{\frac{1}{2}} \tilde{A}=A^{+} \mathrm{e}^{\mathrm{i} \frac{1}{4} \pi}+B^{+} \mathrm{e}^{-\mathrm{i} \frac{1}{4} \pi},
$$




$$
B^{-}=\left(\frac{\chi}{\pi k_{0}}\right)^{\frac{1}{2}} \widetilde{B}=A^{+} \mathrm{e}^{-\mathrm{i} \frac{1}{4} \pi}+B^{+} \mathrm{e}^{1 \frac{1}{4} \pi}
$$

which ensure matching between the limiting forms in (51).

Applying the outgoing-wave condition (14) to (51) shows that $B^{+}=0$. The corresponding solution can then be written as

$$
v_{1}(r) \sim Q_{n}^{-\frac{1}{2}}(r) A_{1}^{+} \mathrm{e}^{\mathrm{i} k_{0} \zeta_{n}(r)}
$$

as $k_{0} \rightarrow \infty$, where attention is restricted to the $r-r_{\delta} \gg k_{0}^{-2 / 3}$ behavior since that is all that is required in (20).

The bounded condition (15) requires that $B^{-}=0$. In this case, only the value of the corresponding solution at $r=r_{s}$ appears in (20). However, the location of the turning point $r_{\delta}$ relative to $r_{s}$ varies with both $\theta$ and $n$ and it is therefore convenient to express the $v_{2}$ solution in the uniformly valid composite form

$$
v_{2}\left(r_{s}\right) \sim A_{2}^{-}\left[4 \pi \frac{\sqrt{-\eta_{n}\left(r_{s}\right)}}{Q_{n}\left(r_{s}\right)}\right]^{\frac{1}{2}} \operatorname{Ai}\left[\eta_{n}\left(r_{s}\right)\right],
$$

as $k_{0} \rightarrow \infty$, where

$$
\eta_{n}(r) \equiv-\left[\frac{3}{2} k_{0} \zeta_{n}(r)\right]^{\frac{2}{3}}
$$

with the cube root defined such that $\eta_{n} \lessgtr 0$ for $r \gtrless r_{j}$.

Since the Wronskian $V$ is independent of $r$, the $r-r_{\delta} \gg k_{0}^{-2 / 3}$ behavior of $v_{1}$ and $v_{2}$ can be used to show that

$$
V \equiv v_{1} v_{2}^{\prime}-v_{1}^{\prime} v_{2} \sim-\mathrm{i} 2 k_{0} A_{1}^{+} A_{2}^{-} \mathrm{e}^{\mathrm{i} \frac{1}{4} \pi}
$$

as $k_{0} \rightarrow \infty$. Combining this result with the expressions for $v_{1}(r)$ and $v_{2}\left(r_{s}\right)$ given above leads to

$$
\frac{v_{1}(r) v_{2}\left(r_{s}\right)}{V} \sim\left[\frac{\mathrm{i} \pi}{k_{0}^{2}} \frac{\sqrt{-\eta_{n}\left(r_{s}\right)}}{Q_{n}\left(r_{s}\right) Q_{n}(r)}\right]^{\frac{1}{2}} \operatorname{Ai}\left[\eta_{n}\left(r_{s}\right)\right] \mathrm{e}^{\mathrm{i} k_{0} \zeta_{n}(r)}
$$

as $k_{0} \rightarrow \infty$.

\section{B Quasi-symmetric, high-frequency approximation}

In this appendix, the high-frequency solutions to (13) are constructed using the azimuthal wavenumber scaling $n=O(1)$. Equations (16) and (17) show that $k_{0}^{2} Q_{n}^{2}$ and $S$ then become of equal order as $r$ becomes sufficiently small. The disordering of (13) is dealt with, as in reference [2], by introducing an inner region where

$$
\bar{r} \equiv k_{0} r=O(1)
$$

The corresponding equation for $v$ is given to the required order of accuracy by

$$
\bar{r}^{2} \frac{\mathrm{d}^{2} v}{\mathrm{~d} \bar{r}^{2}}+\left[\bar{r}^{2} q^{2}(0)-n^{2}+\frac{1}{4}\right] v=0
$$

which has the general solution

$$
v \sim \sqrt{\bar{r}}\left[\bar{A} \mathbf{J}_{n}(\bar{\xi})+\bar{B} \mathbf{H}_{n}^{(1)}(\bar{\xi})\right]
$$

as $k_{0} \rightarrow \infty$, where $\bar{\xi} \equiv q(0) \bar{r}$ and $\mathrm{J}_{n}$ and $\mathrm{H}_{n}^{(1)}$ denote the Bessel and Hankel functions of the first kind, respectively.

When $r$ is order one, the solution to (13) is determined, to the required order of accuracy, by

$$
v^{\prime \prime}+k_{0}^{2} q^{2} v=0
$$

which can, of course, be solved using WKB theory [11]. The particular form of the solution depends on the number and nature of the turning points determined by the zeroes of $q^{2}$. As in Appendix A, it will be assumed here, for simplicity, that the $v$ equation has at most one simple turning point. 
First, suppose that (56) has a turning point at $r=r_{\sigma} \gg 1 / k_{0}$ where the notation $r_{\sigma}$ is used to distinguish the zeroes of $q^{2}$ from the $n$-dependent zeroes of $Q_{n}^{2}$. The solution to (56) is then given by (51), (52) and (53) with $n$ set equal to zero. Matching that result with (55) as $\bar{r} \rightarrow O\left(k_{0}\right)$ (noting that $\bar{\xi}$ is purely imaginary) requires

$$
\sqrt{2 \pi} A^{-}=\mathrm{e}^{-\mathrm{i}\left(k_{0} \xi_{\sigma}-\frac{1}{2} n \pi\right)} \bar{A}, \quad \sqrt{2 \pi} B^{-}=-\mathrm{i} 2 \mathrm{e}^{\mathrm{i}\left(k_{0} \xi_{\sigma}-\frac{1}{2} n \pi\right)} \bar{B},
$$

where

$$
\xi(r) \equiv \int_{0}^{r} q(r) \mathbf{d} r
$$

and a subscript $\sigma$ is used to indicate evaluation at $r=r_{\sigma}$.

Applying the outgoing-wave condition (14) shows that $B^{+}=0$ from which follows

$$
v_{1}(r) \sim A_{1}^{+}\left(\frac{\mathrm{i} \pi}{2} \frac{k_{0} \xi}{q}\right)^{\frac{1}{2}} \mathrm{H}_{n}^{(1)}\left(k_{0} \xi\right) \mathrm{e}^{-1\left(k_{0} \xi_{\sigma}-\frac{1}{2} n \pi\right)},
$$

as $k_{0} \rightarrow \infty$, where attention is restricted to the $r-r_{\sigma} \gg k_{0}^{-2 / 3}$ behavior since that is all that is required in (20) and the Hankel function has been introduced (without loss of generality) in order to facilitate the application of Graf's addition theorem [12] in section 3 .

The bounded condition (15) requires $\bar{B}=0$ and the corresponding expression for $v_{2}\left(r_{s}\right)$ depends on the location of $r_{\sigma}$ relative to $r_{s}$. Reasoning that the quasi-symmetric approximation is only appropriate when $r_{s} \ll 1$, Balsa [2] employed the simplifying assumption that $r_{\sigma}-r_{s} \gg k_{0}^{-2 / 3}$, i.e. that the source is always closer to the jet axis than the turning point. Using this assumption, $v_{2}\left(r_{s}\right)$ can be expressed as

$$
v_{2}\left(r_{s}\right) \sim \bar{A}_{2}\left(\frac{k_{0} \xi_{s}}{q_{s}}\right)^{\frac{1}{2}} \mathrm{~J}_{n}\left(k_{0} \xi_{s}\right),
$$

as $k_{0} \rightarrow \infty$. It should be noted that for any given value of $r_{s}$ there will be, in general, a range of $\theta$ for which $r_{s}>r_{\sigma}$ and, consequently, for which the above expression is invalid. The implications of this failure are discussed in section 4 where comparisons with the order-one frequency solution for the reduced Green's function are given.

Unlike the situation encountered in Appendix $\mathrm{A}$, it is possible, with the present scaling of $n$, that the equation governing $v$ has no turning points. When this is the case, the solution to (56) is given as

$$
v \sim q^{-\frac{1}{2}}\left(A^{+} \mathrm{e}^{1 k_{0} \zeta_{0}}+B^{+} \mathrm{e}^{-\mathrm{i} k_{0} \zeta_{0}}\right),
$$

as $k_{0} \rightarrow \infty$. Matching with (55) as $\bar{r} \rightarrow O\left(k_{0}\right)$ (noting that $\bar{\xi}$ is now purely real) requires

$$
\sqrt{\mathrm{i} 2 \pi} A^{+}=(\bar{A}+2 \bar{B}) \mathrm{e}^{-\mathrm{i} \frac{1}{2} n \pi}, \quad \sqrt{\mathrm{i} 2 \pi} B^{+}=\mathrm{i} \bar{A} \mathrm{e}^{\mathrm{i} \frac{1}{2} n \pi} .
$$

Expressions for $v_{1}(r)$ and $v_{2}\left(r_{s}\right)$ can be derived by applying the boundary conditions (14) and (15) as done above. It turns out that the final expressions are in exact agreement with those given by (57) and (58) if the convention that $r_{\sigma}=0$ when $q^{2}$ has no zeroes is adopted.

The $r$-independent Wronskian $V$ can be evaluated using the $r-r_{\sigma} \gg k_{0}^{-2 / 3}$ behaviors of $v_{1}$ and $v_{2}$ and is given, for the one- and no-turning point solutions, by

$$
V \equiv v_{1} v_{2}^{\prime}-v_{1}^{\prime} v_{2} \sim-\mathrm{i} k_{0}\left(\frac{\mathrm{i} 2}{\pi}\right)^{\frac{1}{2}} A_{1}^{+} \bar{A}_{2} \mathrm{e}^{-\mathrm{i}\left(k_{0} \xi_{\sigma}-\frac{1}{2} n \pi\right)},
$$

as $k_{0} \rightarrow \infty$. Combining this result with the expressions for $v_{1}(r)$ and $v_{2}\left(r_{s}\right)$ given above leads to

$$
\frac{v_{1}(r) v_{2}\left(r_{s}\right)}{V} \sim \frac{\mathrm{i} \pi}{2}\left(\frac{\xi_{s} \xi}{q_{s} q}\right)^{\frac{1}{2}} \mathrm{H}_{n}^{(1)}\left(k_{0} \xi\right) \mathbf{J}_{n}\left(k_{0} \xi_{s}\right)
$$

as $k_{0} \rightarrow \infty$. 


\section{Ray-theory approximation}

In this appendix, the high-frequency solution to (4) obtained from ray theory is reviewed. Following the matched asymptotic analysis given by Durbin [4], the solution to (4) for the uni-directional, transversely sheared mean flow (1) is

$$
G_{\omega}\left(\boldsymbol{x} \mid \boldsymbol{x}_{s}\right) \sim \Phi(\boldsymbol{x}) A\left(\boldsymbol{x} \mid \boldsymbol{x}_{s}\right) \mathrm{e}^{1 k_{0} S\left(\boldsymbol{x} \mid \boldsymbol{x}_{s}\right)},
$$

as $k_{0} \rightarrow \infty$, where the Eikenol $S$ satisfies

$$
\Phi^{2}-|s|^{2}=0, \quad s \equiv \nabla S
$$

the amplitude function $A$ satisfies

$$
\nabla \cdot\left[\left(s+i \frac{M}{a} \Phi\right) A^{2}\right]=0
$$

and here $\Phi \equiv(1-M i \cdot s) / a$.

The first-order partial-differential equation (61) is reduced to the coupled system of ordinary differential equations,

$$
\dot{x}=s+i \frac{M}{a} \Phi, \quad \dot{s}=\frac{1}{2} \nabla_{\perp}\left(\Phi^{2}\right), \quad \dot{S}=s \cdot \dot{x},
$$

along the rays $\boldsymbol{x}(\tau)$ by the method of characteristics, where $\tau$ is a parameter that varies continuously along the ray, a dot indicates differentiation with respect to $\tau$ and $\nabla_{\perp}$ is the gradient operator in the $y-z$ plane. Equations (63) must be solved subject to initial conditions at the source,

$$
\boldsymbol{x}=x_{s}, \quad \dot{\boldsymbol{x}}=\sigma_{s}\{\cos \mu, \sin \mu \cos \lambda, \sin \mu \sin \lambda\}, \quad S=0,
$$

at $\tau=0$, where the free parameters $\mu$ and $\lambda$ determine the initial ray direction relative to the Cartesian coordinate system $\{x, y, z\}$ and it follows from (61) and (63) that

$$
\sigma_{s}^{-2}=a_{s}^{2}-M_{s}^{2} \sin ^{2} \mu .
$$

The amplitude function $A$ is found by solving (62) subject to matching with a near-source solution. It follows from the analysis of reference [4] that

$$
A\left(\boldsymbol{x} \mid \boldsymbol{x}_{s}\right)=\frac{1}{4 \pi a_{s} \Phi_{s}}\left(\frac{\sigma_{s}^{3} \sin \mu}{J}\right)^{\frac{1}{2}}
$$

where

$$
J=\frac{\partial(x, y, z)}{\partial(\tau, \mu, \lambda)}
$$

is the Jacobian determinant.

When attention is restricted to the far-field behavior, the solution (60) can be simplified by noting that the rays $x(\tau)$ become straight lines in the absence of a mean flow. Thus, in the far field, the approximation,

$$
\boldsymbol{x} \sim \boldsymbol{x}_{s}+R_{\infty}\left\{\cos \theta_{\infty}, \sin \theta_{\infty} \cos \phi_{\infty}, \sin \theta_{\infty} \sin \phi_{\infty}\right\}
$$

can be introduced, where $R_{\infty}$ is the distance between the far-field observation point and the source point and $\theta_{\infty}$ and $\phi_{\infty}$ are the far-field polar and azimuthal angles measured from axes passing through the source point and aligned with the $x$ and $y$ directions, respectively. It is important to note that $\phi_{\infty}, \theta_{\infty}$ and $R_{\infty}$ are not equal to $\varphi, \theta$ and $R$ of sections 2 and 3 but approach these quantities in the far field, i.e.

$$
\begin{gathered}
\phi_{\infty} \sim \varphi+\frac{r_{s}}{R} \csc \theta \sin \Delta \varphi+\cdots, \\
\theta_{\infty} \sim \theta+\frac{r_{s}}{R} \cos \theta(1-\cos \Delta \varphi)+\cdots, \\
R_{\infty} \sim R+r_{s} \sin \theta(1-\cos \Delta \varphi)+\cdots,
\end{gathered}
$$


as $R \rightarrow \infty$.

It follows from (61) and (63) that $\dot{R}_{\infty}=1$ which can then be used when inserting (68) into (67) to obtain

$$
J \sim R_{\infty}^{2} \sin \theta_{\infty} \frac{\partial\left(\theta_{\infty}, \phi_{\infty}\right)}{\partial(\mu, \lambda)}
$$

as $R_{\infty} \rightarrow \infty$. Since (63) implies that the quantity $i \cdot s$ is constant along each ray, (64) and (68) show that

$$
i \cdot s=\frac{a_{s}^{2} \sigma_{s} \cos \mu-M_{s}}{a_{s}^{2}-M_{s}^{2}}=\cos \theta_{\infty},
$$

and furthermore, in view of $(65), \theta_{\infty}=\theta_{\infty}(\mu)$ and

$$
\sin \theta_{\infty} \frac{\mathbf{d} \theta_{\infty}}{\mathbf{d} \mu}=a_{s}^{2} \sigma_{s}^{3} \sin \mu
$$

An additional consequence of (73) is

$$
\sigma_{s}^{2} \sin ^{2} \mu=\Phi_{s}^{2}-\cos ^{2} \theta_{\infty},
$$

which follows directly from the Eikenol equation (61). The Eikenol itself has the far-field behavior

$$
S \sim R_{\infty}+S_{\perp}\left(\boldsymbol{x} \mid x_{s}\right)
$$

as $R_{\infty} \rightarrow \infty$, where

$$
S_{\perp} \equiv \int_{0}^{\tau}\left(s \cdot \dot{x}_{\perp}-\sin ^{2} \theta_{\infty}\right) \mathrm{d} \tau
$$

remains bounded as $R_{\infty} \rightarrow \infty$ and $x_{\perp}=\{y, z\}$ denotes the ray vector in the $y-z$ plane.

Substituting (74) into (72) and the result into (66) yields the far-field approximation for $A$ which when substituted, together with (76), into (60) leads to (18) and (19) where now

$$
\mathcal{R}_{\omega} \sim\left(\frac{\partial \varphi}{\partial \lambda}\right)^{-\frac{1}{2}} \mathrm{e}^{\mathrm{i} k_{0}\left(S_{\perp}+R_{\infty}-R\right)},
$$

as $k_{0}, R \rightarrow \infty$, and use has been made of the far-field relations (69)-(71).

For the purposes of the present investigation, it is convenient to restate (63) and (64) in terms of the cylindrical coordinates $\{x, r, \varphi\}$ of section 2 . The ray equations in the $y-z$ plane are then

$$
\left.\begin{array}{ll}
(\dot{r})^{2}=\Phi^{2}-\cos ^{2} \theta_{\infty}-s^{(\varphi)}, & r \dot{\varphi}=s^{(\varphi)} \\
\left(r s^{(\varphi)}\right)=\frac{1}{2} \partial\left(\Phi^{2}\right) / \partial \varphi, & \dot{S}_{\perp}=r s^{(\varphi)} \dot{\varphi}+(\dot{r})^{2}-\sin ^{2} \theta_{\infty},
\end{array}\right\}
$$

which must be solved subject to

$$
r=r_{s}, \quad \varphi=\varphi_{s}, \quad s^{(\varphi)}=\sigma_{s} \sin \mu \sin \left(\lambda-\varphi_{s}\right), \quad S_{\perp}=0,
$$

at $\tau=0$, where $r s^{(\varphi)} \equiv \partial S / \partial \varphi$. 
Public reporting burden for this collection of information is estımated to average 1 hour per response, including the time for reviewing instructions, searching existing data sources, gathering and maintainıng the data needed, and completıng and reviewing the collection of information. Send comments regarding this burden estımate or any other aspect of this collection of information, including suggestions for reducing this burden, to Wastington Headquarters Services. Directorate for Information Operations and Reports, 1215 Jefferson Davis Highway, Sute 1204, Arlington, VA 22202-4302, and to the Office of Management and Budget, Paperwork Reduction Project (0704-0188), Washington, DC 20503

1. AGENCY USE ONLY (Leave blank) 2. REPORT DATE 4. TITLE AND SUBTITLE January 2003 3. REPORT TYPE AND DATES COVERED Final Contractor Report

On the Applicability of High-Frequency Approximations to Lilley's Equation 6. AUTHOR(S)

WBS-22-708-87-25 GESS-005

David W. Wundrow and Abbas Khavaran

\section{PERFORMING ORGANIZATION NAME(S) AND ADDRESS(ES)}

Ohio Aerospace Institute 22800 Cedar Point Road

Brook Park, Ohio 44142
5. FUNDING NUMBERS
9. SPONSORING/MONITORING AGENCY NAME(S) AND ADDRESS(ES)

National Aeronautics and Space Administration

Washington, DC 20546-0001
8. PERForming oRganization REPORT NUMBER

E-13742

10. SPONSORING/MONITORING AGENCY REPORT NUMBER

NASA CR-2003-212089

11. SUPPLEMENTARY NOTES

David W. Wundrow, Ohio Aerospace Institute, Brook Park, Ohio 44142, and Abbas Khavaran, QSS Group Inc., Cleveland, Ohio 44135. Project Manager, James Bridges, Structures and Acoustics Division, NASA Glenn Research Center, organization code 5940, 216-433-2693.

12a. DISTRIBUTION/AVAILABILITY STATEMENT

Unclassified - Unlimited

Subject Category: 71
Distribution: Nonstandard

Available electronically at http://gltrs.grc.nasa.gov

This publication is available from the NASA Center for AeroSpace Information. 301-621-0390.

\section{ABSTRACT (Maximum 200 words)}

Three forms of the high-frequency asymptotic Green's function for Lilley's equation are reviewed and compared to the exact solution over wide range of Strouhal numbers. The asymmetric approximation, which applies to sources away form the jet axis, and the quasi-symmetric approximation, which is arrived at by making a near-axis source assumption, are both obtained for parallel round jets from a formal Fourier-transform solution. The ray-theory solution, which is the only high-frequency approximation that can be applied to more general mean flows, follows from a WKB ansatz and is shown to be closely related to the asymmetric approximation. The comparisons show that the best overall prediction of the exact Green's function is given by the asymmetric approximation which remains accurate down to a Strouhal number of 1/2. The close relationship between the asymmetric and ray-theory approximations suggests that the highfrequency asymptotic Green's function for more general mean flows would be similarly successful.

\begin{tabular}{|c|c|c|}
\hline $\begin{array}{l}\text { 14. SUBJECT TERMS } \\
\text { Aeroacoustics; Asymptot } \\
\text { flow; Noise prediction; } R\end{array}$ & $\begin{array}{l}\text { roperties; Geometrical } \\
\text { action }\end{array}$ & $\mathrm{Gr}$ \\
\hline $\begin{array}{l}\text { 17. SECURITY CLASSIFICATION } \\
\text { OF REPORT } \\
\text { Unclassified }\end{array}$ & $\begin{array}{l}\text { 18. SECURITY CLASSIFICATION } \\
\text { OF THIS PAGE } \\
\text { Unclassified }\end{array}$ & $\begin{array}{l}\text { 19. SECURITY CLASSIFICATION } \\
\text { OF ABSTRACT } \\
\text { Unclassified }\end{array}$ \\
\hline
\end{tabular}

\section{NUMBER OF PAGES}

16. PRICE CODE

20. LIMITATION OF ABSTRACT 